\title{
Comparison of the metabolic profiles of rice cultivars with different eating qualities during grain development and the post-harvesting process
}

\section{Yi-Bo Chen}

Guangdong Academy of Agricultural Sciences

\section{zhidong wang}

Guangdong Academy of Agricultural Sciences

chongrong wang

Guangdong Academy of Agricultural Sciences

Hong Li

Guangdong Academy of Agricultural Sciences

\section{Daoqiang Huang}

Guangdong Academy of Agricultural Sciences

\section{Degui Zhou}

Guangdong Academy of Agricultural Sciences

\section{Yukun Wu}

Guangdong Academy of Agricultural Sciences

\section{Lei Zhao}

Guangdong Academy of Agricultural Sciences

\section{Rong Gong}

Guangdong Academy of Agricultural Sciences

\section{Yangyang Pan}

Guangdong Academy of Agricultural Sciences

Shaochuan Zhou (Dxx123@163.com)

Guangdong Academy of Agricultural Sciences https://orcid.org/0000-0002-8079-4183

\section{Original article}

Keywords: rice, eating quality, nutritional quality, post-harvesting process, metabolomics

Posted Date: May 29th, 2020

DOI: https://doi.org/10.21203/rs.3.rs-31258/v1 
License: (c) (i) This work is licensed under a Creative Commons Attribution 4.0 International License. Read Full License 
1 Comparison of the metabolic profiles of rice cultivars with different eating

2 qualities during grain development and the post-harvesting process

3 Yi-Bo Chen ${ }^{1,2} \#$, Zhidong Wang ${ }^{1,2} \#$, Chongrong Wang ${ }^{1,2}$, Hong $\mathrm{Li}^{1,2}$, Daoqiang Huang ${ }^{1,2}$,

4 Degui Zhou ${ }^{1,2}$, Yukun $\mathrm{Wu}^{1,2}$, Lei Zhao ${ }^{1,2}$, Rong Gong ${ }^{1,2}$, Yangyang Pan $^{1,2}$, Shaochuan $5 \mathrm{Zhou}^{1,2} *$

$6{ }^{1}$ Guangdong Academy of Agricultural Sciences, Rice Research Institute, Guangzhou, 510640,

7 China.

$8{ }^{2}$ Guangdong Key Laboratory of New Technology in Rice Breeding, Guangzhou, 510640,

9 China.

10 chenyibo1213@126.com (Y.C.); zhidongwang@163.com (Z.D.); wangcr1980@163.com

11 (C.W.); gdxxs123@126.com (H.L.); gzhdq1@163.com (D.H.); zhoudegui@126.com (D.Z.);

12 wuyukun68@163.com (Y.W.); zhaoleijx@163.com (L.Z.); gongrong2008@126.com (G.R.);

13 yangyangpan@126.com (Y.P.)

14 \#These authors contributed equally to this work.

15 *Correspondence Tel +86 20 87588589; fax +86 20 87588589; email xxs123@163.com.

16

17 
19 Background: Rice eating quality and nutritional quality is affected by both grain 20 development and the post-harvesting process, but the dynamic metabolite changes 21 among rice cultivars during these processes are unclear.

22 Results: We successfully identified 623 metabolites in four indica cultivars with 23 different eating qualities using a widely-targeted metabolomics approach, and found 24 that the metabolic variation became increasingly smaller and differed according to the 25 stage and cultivar during grain development and post-harvesting. Our results 26 suggested that the levels of sugars, amino acids, lipids, and flavones demonstrated 27 cultivar-specific changes during grain development, and sufficient carbon supply 28 during grain development may contribute to the formation of excellent eating quality.

29 We further found that most of the metabolites, especially the nutritional metabolites, 30 decreased significantly or even lost, whereas only few increased during post31 harvesting, further indicating that the post-harvesting process reduced the metabolic 32 molecular differences between rice cultivars with different eating qualities, and had an 33 even greater influence on metabolites than genetic factors.

34 Conclusions: We found that a large number of metabolites changed significantly in 35 the rice cultivars during grain development and the post-harvesting process, which 36 facilitates further study of rice quality formation and has potential application in 37 eating quality and nutritional quality improvement.

38 Keywords: rice, eating quality, nutritional quality, post-harvesting process, 39 metabolomics 


\section{Background}

41 Rice has been consumed for almost 5000 years and currently feeds almost half of the

42 human population (Liu et al. 2013). With the improvement in living standards, rice quality is now a top consideration for customers and breeding programs. Rice quality is mainly related to grain appearance, milling quality, nutritional quality, and cooking and eating quality (Hori 2018). In addition, rice cultivars of different qualities are also used for medical, ceremonial, or special production purposes (Tian et al. 2009).

Rice quality, especially the eating quality and nutritional quality, is closely related to the chemical composition of the grains, including starch (mainly amylose and amylopectin), proteins, and lipids. These metabolites are synthesized and genetically regulated during rice grain development. The development of the rice grain can be divided into three basic stages: the embryonic development stage, the cell division stage, and the morphogenesis stage; the second stage of grain maturation, during which a large number of storage reserves accumulate; and the third stage of grain drying and dormancy (Deng and Wang 2013; Sreenivasulu et al. 2015). These three stages are associated with substantial spatiotemporal metabolic rearrangements regulated by global gene expression programs. Previous studies mainly focused on the

57 mechanisms of rice quality formation at the level of gene regulation. For example, 58 Zhao et al. found that GS9 acts as a transcriptional activator to regulate rice grain 59 shape and appearance (Zhao et al. 2018). It is reported that the natural variation in 60 OsGluA2 is involved in grain protein content regulation in rice (Yang et al. 2019). 61 Wang et al. reported that a lipid transfer protein, OsLTPL36, is essential for grain 62 development and grain quality in rice (Wang et al.2015). However, few studies have 
63 investigated the mechanism behind the formation of rice quality at the global metabolite level.

With the exception of genetic regulation during grain development, rice quality, especially the eating quality and nutritional quality, is partially affected by the postharvesting processes, which can cause physical, chemical, and structural changes in the grain (Corrêa et al. 2007). The post-harvesting processes, including harvesting, threshing, drying, transportation, storage, rice processing, and milling, are primarily influenced by human factors rather than genetic regulation. During these processes, harvesting at the incorrect product maturity stage, excessive exposure to rain and extreme temperatures, and microbial contamination can seriously affect rice quality (Taiwo and Bart 2016). For example, rice milling is a crucial step whereby the husk and the bran layers (including cuticular layer and aleurone layer embryo) are removed. During this process, a large amount of nutritional metabolites, such as sterols, $\gamma$ oryzanols, tocopherols, tocotrienols, and phenolic compounds, is lost in the rice bran, which is a processing byproduct (Aguilar et al. 2007). However, few studies have aimed to clarify how many beneficial metabolites are retained in the polished rice to be consumed during the post-harvesting processes. Studying the metabolites remaining in polished rice can improve the quality of rice, especially the nutritional

81 quality. For example, Zhang et al. (2019) reported that plant metabolic engineering and synthetic biology strategies can be effectively and accurately applied in the synthesis of specific micronutrients, phytonutrients, and/or bioactive components in crops.

Finally, regardless of gene regulation during grain development or artificial factors during the post-harvesting processes, the quality of rice is closely related to its 
metabolites. It is thus of great significance that the changes in metabolites during grain development and the post-harvesting are explored to contribute to rice quality and breeding research. Metabolomics has provided an efficient large-scale solution that complements traditional and genomic approaches for investigating rice grain development and the post-harvesting processes (Chen et al. 2016; Sulpice 2019; Zhu et al. 2018). In this study, a widely targeted metabolomics was employed to elucidate the kinetic metabolic changes along the grain development and the post-harvesting processes in four indica cultivars with different eating qualities. Our results showed that the levels of a large number of metabolites changed significantly in the four rice cultivars during grain development and the post-harvesting process. Our findings provide a foundation for studies on rice quality formation and have potential application in rice quality improvement.

\section{Results}

\section{Eating Quality Values of the Four Indica Cultivars}

To investigate the changes in metabolites during grain development and the postharvesting process, we selected four indica cultivars from a pedigree with different eating qualities: MXZ (MeiXiangZhan2), MSZ (MeiSiZhan), HHZ (HuangHuaZhan), and QXZ (QiXinZhan) (Figs. 1a and S1). The eating quality value results showed that MXZ, the largest conventional rice planted in Guangdong province of China at present, has the highest eating quality of 88 . The eating qualities of HHZ, grown in over 4.5 million ha in southern China (Zhou et al. 2016), and MSZ were 86 and 84, respectively, while that of QXZ (negative control) was only 64.7 (Fig. 1a).

\section{Overview of the Metabolic Profiles of All Rice Samples}


110 In order to investigate the changes in metabolites during rice grain development and

111 the post-harvesting process, samples of grains at 8, 15, 30, and 40 DAF were collected.

112 Samples at 40 DAF were polished rice that had been dried and milled and lacked the

113 endosperm and aleurone layers. These were collected in order to investigate the 114 metabolite changes during the post-harvesting process and were subjected to 115 metabolic profiling analysis using LC-ESI-MS/MS. A total of 623 metabolites were 116 identified, including 95 amino acids and derivatives, 85 organic acids and derivatives, 11767 lipids, 62 flavones, and 58 nucleotides and derivates (Fig. 1b and Table S1). PCA was subsequently performed on the 623 metabolites to visualize the 119 kinetic metabolome patterns of the developing rice grains. The developing rice grains 120 of the four cultivars showed a similar dynamic pattern in the changes in their 121 metabolomes (Fig. 1c). During the grain-filling stage at 8 DAF, there was clear and 122 broad separation of the different cultivars. During the grain-desiccation stage from 15 123 to $30 \mathrm{DAF}$, the separation between the samples of $15 \mathrm{DAF}$ and those of $30 \mathrm{DAF}$ was 124 almost indistinguishable. During the later stage at $40 \mathrm{DAF}$, the separation among the 125 cultivars was smallest compared to those at 8, 15, and 30 DAF. These data indicated 126 that as the grain develops, the metabolic variation among the different cultivars 127 becomes smaller.

As PCA is not able to distinguish the exact contribution of each variable to the 129 observed variation, two-way ANOVA (analysis of variance) and ASCA (ANOVA130 simultaneous component analysis) were then conducted to deconstruct the metabolic 131 variations derived from stage, cultivar, and their interaction. The abundances of 454, 132588 , and 498 metabolites were significantly affected by stage, cultivar, and their 133 interaction, respectively (Figs. 1d, S2, and S3). Among these, the abundances of 623 
metabolites were simultaneously affected by stage, cultivar, and their interaction. In addition, ASCA revealed that $65.13 \%, 40.27 \%$, and $49.78 \%$ of observed metabolic variations could be explained by developmental stage, cultivar, and their interaction, respectively (Figs. S2, and S3). Developmental stage score plots based on PC1 of the corresponding submodels showed that the scores gradually decreased from 8 to 40 DAF, which is consistent with the PCA result shown in Fig. 1, indicating that the metabolomes of the different cultivars had shifted to the same direction with time.

Cultivar score plots showed that HHZ and MSZ had the highest and the lowest score, respectively (Fig. S3). The interaction score plot of HHZ decreased from 8 to $30 \mathrm{DAF}$ and increased at $30 \mathrm{DAF}$, whereas those of MSZ and QXZ demonstrated the opposite trend. The interaction score plot of MXZ increased continuously from 8 to $40 \mathrm{DAF}$.

145 Furthermore, leverage/squared prediction error (SPE) plots were produced to correlate 146 the metabolic features with the experimental factors. Leverage/SPE analysis revealed 147 that the numbers of identified metabolomes responsible for the observed variations 148 derived from time, cultivar, and their interaction were 32,16 , and 36 , respectively 149 (Tables. S2-4).

151 is clear and wide at $8 \mathrm{DAF}$ and becomes smaller from 15 to $40 \mathrm{DAF}$, and the 152 metabolites among the cultivars were affected by stage and cultivar.

\section{Carbohydrate Metabolism During Rice Grain Development}

154 Starch, an end product of photosynthesis in source tissues, is stored as energy reserves 155 in the rice grain and is composed of two major components, namely amylose and 156 amylopectin (Wang et al. 2013). Six of eight metabolites present in the starch 157 biosynthesis pathway were identified herein (Fig. 2a). We found that the levels of 
158

159

160

161

162

163

164

165

166

167

168

169

170

171 gradually decreased from 8 to15 DAF. The levels of G1P in MXZ and HHZ gradually

172 decreased from 8 to $15 \mathrm{DAF}$. These results indicated that the levels of metabolites of

173 starch biosynthesis exhibit cultivar-specific changes, such as F6P, G6P, and G1P in

174 HHZ and MXZ. Furthermore, sufficient carbon supply from the leaf during grain

175 development may contribute to the formation of excellent eating quality.

176 We compared the changes in the 19 carbohydrates identified among the four

177 cultivars during grain development (Fig. 2b). Most significantly, we found that the

178 levels of $57.9 \%$ (11 of 19) of carbohydrates demonstrated similar change patterns in

179 MSZ and QXZ during the grain development process. Three carbohydrates exhibited

180 cultivar-specific changes, including $\mathrm{N}$-acetyl-D-glucosamine and threose in HHZ and 
181 glucosamine in MXZ. We further found that the most significant difference in 182 carbohydrates between the rice cultivars was at 8 DAF (Fig. 2c). We found that $10.5 \%$ 183 of carbohydrates (two of 19) were significantly higher in HHZ than in MXZ, whereas $18442.1 \%$ (eight of 19) were lower in MSZ and QXZ than in MXZ at 8 DAF (Table. S5). 185 Additionally, 5.3\% of carbohydrates (one of 19) were significantly higher in HHZ 186 than in MXZ, whereas 21\% (four of 19) were lower in MSZ than in MXZ at 30 DAF 187 (Table. S6).

\section{Amino Acid and Derivatives Metabolism During Grain Development}

189 Amino acids are primarily utilized for the synthesis of grain-storage proteins, which 190 are important factors affecting the eating quality and nutritional quality of rice and 191 serve as precursors for the biosynthesis of secondary metabolites and as a source of 192 energy (Amir et al. 2018). We identified 95 amino acids and derivatives, which were 193 the most abundant component of the metabolic profiles (Figs. 1b and 3). We found 194 that most amino acids showed cultivar-specific changes during grain development 195 (from 8 to 30 DAF) (Fig. 3a). The levels of 36 amino acids in HHZ, 12 amino acids in 196 MXZ, 11 amino acids in MSZ, and 6 amino acids in QXZ specificity increased 197 significantly at 15 and $30 \mathrm{DAF}$ as compared with those at $8 \mathrm{DAF}$. For example, the 198 levels of L-tyramine in HHZ only increased significantly at 15 and $30 \mathrm{DAF}$ as 199 compared with that at 8 DAF.

We further compared the changes in the amino acids and derivatives identified in

201 HHZ, MSZ, and QXZ with those in MXZ during grain development (Fig. 3b). We 202 found that the most significant difference in amino acids between the rice cultivars 
204 lower than in MXZ, whereas $14.7 \%$ (14 of 95) were lower in QXZ than in MXZ at 8

205 DAF (Table. S7). Furthermore, 26.3\% (25 of 95) of amino acids were significantly 206 higher in HHZ than in MXZ, whereas 24.2\% (23 of 95) were lower in QXZ than in 207 MXZ at 15 DAF (Table. S8). A total of $8.4 \%$ of amino acids (8 of 95) were 208 significantly lower in HHZ than in MXZ, whereas 29.5\% (28 of 95) were lower in 209 MSZ at 30 DAF (Table. S9). In addition, we further investigated eight types of essential amino acids in the 211 four cultivars (Fig. S4). Except for L-threonine, the amino acids in MXZ, QXZ, and 212 HHZ exhibited the same trend, and the levels of the amino acids in MXZ were always 213 higher than those in the other two cultivars from 8 to 30 DAF. In addition, with the 214 exception of L-tryptophan and L-threonine, the levels of the amino acids in HHZ 215 demonstrated completely opposite trends from those in the other three cultivars, 216 possibly due to the genetic relationship of the rice cultivars.

\section{Lipid Metabolism During Grain Development}

218 Storage lipids are vital components for maintaining the structure of grain storage 219 substances and are valuable for rice eating quality and food texture, despite that lipids 220 are only a minor nutrient compared to starch and protein. A total of 66 lipids were 221 identified herein, including 34.8\% (23 of 66) lysophosphatidylcholine (LysoPCs), $22212.1 \%$ (eight of 66) lysophosphatidylethanolamine (LysoPEs), 13.6\% (nine of 66) 223 MAGs (monoacylglycerols), and 39.4\% (26 of 66) other lipids (Fig. 4). We found that 
224 lipids showed apparent cultivar-specific changes from 8 to 30 DAF. Most notably, the

225 levels of 36 lipids, specificity MAG and LysoPC, increased significantly in HHZ,

226 while those in MXZ decreased. Interestingly, compared with the other three cultivars,

227 we found that the lipids with significant changes in HHZ were most significant only at

2288 DAF. These results indicate that the early stage of rice development is the key

229 period for lipid differentiation.

230 Flavones and Other Metabolites During Grain Development

231 In addition to metabolic molecules related to starch, protein, and lipid synthesis, we

232 also identified many types of metabolites that are closely related to human health,

233 such as flavonoids and vitamins. Flavones play an important role in human health due

234 to their pharmacological properties as nutraceuticals and radical scavengers (Tapas,

235 Sakarkar, \& Kakde, 2008). We identified 62 flavones herein (Fig. 5) and found that

236 the level of 12 and 15 flavones in MXZ and HHZ, respectively, showed cultivar-

237 specific increases during grain development. Interestingly, the levels of the specific

238 flavonoids in QXZ (the negative control) were significantly higher than the other

239 three cultivars at $30 \mathrm{DAF}$, such as tricin, a potential multifunctional nutraceutical

240 (Zhou and Ibrahim 2010).

241 In addition, we identified six vitamins, all of which belong to the vitamin B

242 family (Fig. S5). The levels of vitamin B1 and vitamin B5 exhibited similar trends

243 during grain development in the four cultivars. The levels of the other vitamins in

244 MXZ, MSZ, and QXZ were significantly reduced, while those in HHZ showed

245 completely opposite trends, possibly due to the genetic relationship of the rice 
cultivars.

\section{Changes in Metabolites in Rice Grains During the Post-harvesting Processes}

248 We further explored the changes in rice grain metabolites during the post-harvesting 249 process and found that $49.28 \%-57.14 \%$ (307-356) of metabolites were significantly 250 decreased, while only $1.93 \%-4.98 \%$ (12-31) of metabolites were significantly 251 increased in the four cultivars (Fig. 6a). The metabolites with significant changes in 252 HHZ were the most varied among the four cultivars, showing significant variety 253 specificity. There were 307-358 metabolites (a total of 22 types of metabolites) that 254 were significantly reduced in the rice cultivars during this process, of which the 255 largest proportion was amino acids and derivatives, followed by organic acids and 256 nucleic acids and derivatives in the four cultivars (Fig. S6a). This may be due to the 257 loss of embryos during milling, as embryos are rich in proteins and other substances 258 that facilitate seed germination. We found that most of the nutritional metabolites 259 beneficial to human health were reduced dramatically during processing. For example, 260 the content of eight essential amino acids was significantly reduced by $70 \%-98 \%$, 261 while the content of six vitamins and derivatives was significantly reduced by $76 \%-$ $26291 \%$ in the rice cultivars (Figs. S4 and S5). We also noted that many metabolites 263 beneficial to human health were lost completely in the four cultivars, such as catechin, 264 reported to have anti-cancer, anti-virus, and weight loss functions (Xu et al. 2017). It 265 is worthy to note that the levels of chlorpyrifos, a broad-spectrum and moderately 266 toxic chlorinated organophosphate insecticide used in agricultural production (Chishti 267 et al. 2013), were significantly reduced by 76\%-91\% (Fig. S7). In addition, there 268 were only 12-31 metabolites from a total of 12 types of metabolites that were 269 significantly increased in the cultivars during this process, of which the largest 
proportion was lipids (Fig. S6b). For example, 9-HOTrE and punicic acid in MXZ

271 increased by 3.96 and 4.19 times, respectively.

272 We further analyzed the changes in metabolites between the rice cultivars during 273 this process. We found that at 30 DAF (pre-harvest), the difference between 274 metabolites in HHZ and MXZ was the largest, and the content of 82 and 45 275 metabolites was significantly higher and lower, respectively, while at 40 DAF 276 (polished rice), the difference between them was the smallest, with only 42 277 significantly different metabolites (11 higher levels and 32 lower levels) (Fig. 6b and 278 c). This result suggests that it is possible to reduce the differences in the metabolites 279 of distantly-related rice cultivars during processing. In addition, we analyzed the 280 differences in metabolites between the 40 DAF samples (polished rice)) and found 281 that only 11-27 metabolites in MSZ, HHZ, and QXZ were higher than those in MXZ, 282 while 30-72 metabolites were lower than those in MXZ. Among these, the 283 metabolites that were higher in abundance in MSZ, HHZ, and QXZ mainly included 284 amino acids and nucleotide derivates, while metabolites that were lower in abundance 285 mainly comprised organic acids and derivates in MSZ, flavones in HHZ, and lipids in 286 QXZ (Fig. S8). Interestingly, during this process, although the metabolite differences 287 between the rice cultivars decreased, the unique substances increased at $40 \mathrm{DAF}$. At $28830 \mathrm{DAF}$, there were only four and one unique metabolites in HHZ and QXZ, 289 respectively; after processing, the unique metabolites in MXZ, HHZ, QXZ, and MSZ 290 increased to 11, 8, 17, and 7, respectively (Fig. 6d). Many of these metabolites have 291 medicinal properties; for example, taxifolin (detected in MXZ) was found to exhibit 292 anticancer and neuroprotective effects (Kara et al. 2019), and quinic acid (detected in 
293 QXZ) has been shown to possess radioprotection, anti-neuroinflammatory, and anti294 oxidant activities (Jang et al. 2017; Yan et al. 2018).

\section{Discussion}

296 Metabolites are the final products of cell activities and thus directly reflect rice quality.

297 Profound changes in metabolites occur during grain development and the post298 harvesting processes, and thus understanding the changes in metabolites underlying 299 these processes is important for both a basic understanding of rice biology and for 300 applied rice breeding. Here, our results showed that the levels of a large number of 301 metabolites changed significantly during grain development and the post-harvesting 302 process.

Rice grain development involves the synthesis, interconversion, and accumulation of numerous metabolites to form and accumulate various macromolecules, which directly affects rice eating quality and nutritional quality. The PCA results showed that the metabolic variation among the different cultivars decreased from 8 to 30 DAF (Fig. 1c), which is consistent with previous reports $(\mathrm{Hu}$ et al. 2016). The most clear and wide separation among the cultivars was observed 309 from 8 to 15 DAF, which is the key phase (grain-filling stage) for the accumulation of 310 storage compounds in the grain. Drastic changes in metabolites, including sugars, 311 amino acids, lipids, and flavones, occurred during processing. For example, the levels 312 of sucrose (the main raw material for the synthesis of starch) and UDPG decreased 313 significantly from 8 to 15 DAF in all four cultivars (Fig. 2a). These results indicated 314 that significant changes in these primary and secondary metabolites may be closely 315 related to rice eating quality formation. We further observed that in MXZ (with the 316 best eating quality), the levels of sucrose were higher than in the other three cultivars 
from 8 to 15 DAF (Figs. 2a). These results may indicate that sufficient carbon supply during grain development may contribute to the formation of excellent eating quality.

The most important factor affecting rice quality is the genetic characteristics of the cultivars during grain development. The two-way ANOVA and ASCA results also

321 showed that the abundances of 623 metabolites were simultaneously affected by

322 cultivar, stage, and their interaction (Fig. 1b and d). We found that the levels of sugars, 323 amino acids, lipids, and flavones demonstrated cultivar-specific changes. For example,

324 lipids only increased significantly in HHZ during gain development (Fig. 4a). We also

325 found that the eating quality value of MSZ quality was significantly better than that of

326 QXZ, but the levels of metabolites in the starch biosynthesis pathway (such as glucose, 327 G6P, F6P, and G1P) showed similar change patterns (Fig. 2a). This indicated that the 328 quality of MSZ exhibits varietal specificity in starch synthesis compared with MXZ 329 and HHZ, and that in addition to starch synthesis, other factors significantly affect 330 rice eating quality. It should be noted that although the eating quality of HHZ is 331 significantly better than that of QXZ and similar to that of MXZ and MSZ (Fig. 1a), 332 the changes in some substances showed completely opposite trends. For example, the 333 levels of amino acids, lipids, and vitamins in HHZ demonstrated completely opposite 334 trends from those in the other three cultivars (Figs. 3, 4, and S5), indicating that many 335 metabolite changes show varietal specificity, which is related to the genetic distance 336 between the cultivars. These results indicate that although rice cultivars have similar 337 eating qualities, their metabolites, especially nutrient molecules, differ significantly, 
which provides a theoretical basis for the cultivation of excellent cultivars that

339 balance both taste quality and nutritional quality.

The post-harvesting processes, including harvesting, threshing, drying, 341 transportation, storage, rice processing, and milling, can cause physical, chemical, and 342 structural changes in the grains (Corrêa et al. 2007). The PCA showed that the 343 metabolic variation among the cultivars decreased obviously from 30 (mature grain) 344 to 40 DAF (polished rice) (Fig. 1c), and we found that $49 \%-57 \%$ of the metabolites 345 decreased significantly, whereas only 1\%-4.9\% increased (Fig. 6a). This indicated 346 that there were significant changes in the type and content of metabolites during the 347 process and that the metabolite differences between rice cultivars with different eating 348 qualities were reduced. During this process, the levels of amino acids and their 349 derivatives decreased most significantly, and even many metabolites beneficial to 350 human health were completely lost, such as catechin. These significant changes are 351 likely to be the result of rice milling processing, in which the husk and bran layers 352 (including cortex and embryo) are removed. This is why the rice bran, a by-product of 353 rice processing, contains high amounts of amino acids, lipids, fats, vitamins, and other 354 cofactors that are beneficial to human health (Zarei et al. 2017). Although the levels 355 of most metabolites were reduced or even lost (Fig. 6a), the content of a few 356 substances increases significantly, such as B1 (Fig. S5). We also found that unique 357 metabolites between cultivars increased following processing, such as taxifolin in 358 MXZ (Fig. 6d). Although the content of beneficial metabolites may be low in white 359 rice, it is possible to specifically increase the content of such substances using 360 molecular biology techniques in particular rice cultivars (Zhu et al. 2019). Thus these 361 results suggested that the metabolic differences between the rice varieties were 
reduced, and that most metabolites were significantly reduced, whereas some specific or even unique metabolites were increased during these processes.

In addition, the metabolites with significant changes in HHZ were the most varied among the four cultivars during the post-harvesting process (Fig. 6a), indicating that the metabolite changes are cultivar-specific during the post-harvesting process due to the genetic relationship of the rice cultivars. We found that compared with MSZ and QXZ, the levels of metabolites in HHZ differed most significantly from MXZ at $30 \mathrm{DAF}$, while those in HHZ showed the least difference with MXZ at 40 DAF (Fig. $6 \mathrm{~b}$ and c). This suggested that the post-harvesting process reduced the metabolic differences between the rice cultivars and had an even greater influence on the metabolites than the genetic factors. Finally, by comparing the significantly different metabolites between the 40 DAF samples (white rice), we found that most of the significantly different metabolites were higher in MXZ than in HHZ, MSZ, and QXZ (Fig. 6c), indicating that MXZ is a suitable material for studying rice quality and breeding due to the balance between eating quality and nutritional quality.

In summary, we found that a large number of metabolites changed significantly in the four rice cultivars during grain development and the post-harvesting process. This suggests that studies on rice quality improvement, particularly that of the eating quality and nutritional quality, should consider both the inheritance mechanisms of grain quality formation and factors relating to the post-harvesting process.

\section{Conclusion}

In the present study, 623 metabolites were identified in four indica cultivars from a pedigree with different eating qualities. We found that the metabolic variation became 
increasingly smaller and differed according to the stage and cultivar during grain development and the post-harvesting process. The levels of sugars, amino acids, lipids, and flavones demonstrated cultivar-specific changes during grain development.

388 Sufficient carbon supply during grain development may contribute to the formation of 389 excellent eating quality. We further found that most of the metabolites decreased 390 significantly, whereas only a few increased during the post-harvesting process. These 391 findings provide new insight into rice quality formation and have potential application 392 in quality improvement.

393

394

\section{Measurement of Rice Eating Quality}

404 Seventeen grams of polished rice was washed three times with water. The washed 405 polished rice was placed into a special small cup with $19.04 \mathrm{~g}$ of water and then 406 placed into a rice cooker at $100^{\circ} \mathrm{C}$ and heated for $20 \mathrm{~min}$. Finally, the rice eating 407 quality was measured by the rice taste meter (SATA1B) produced by the Japan Satake 
408 Corporation (Hiroshima, Japan). Each experiment was repeated three times, and data represent the mean \pm standard deviation of three independent experiments.

\section{Sample Preparation and Extraction}

411 The freeze-dried grain was crushed using a mixer mill (MM 400, Retsch) with a 412 zirconia bead for $1.5 \mathrm{~min}$ at $30 \mathrm{~Hz}$. One-hundred milligrams of powder was weighed 413 and extracted overnight at $4^{\circ} \mathrm{C}$ with $1.0 \mathrm{~mL} 70 \%$ aqueous methanol. Following 414 centrifugation at $10,000 \mathrm{~g}$ for $10 \mathrm{~min}$, the extracts were absorbed (CNWBOND 415 Carbon-GCB SPE Cartridge, $250 \mathrm{mg}, 3 \mathrm{~mL}$; ANPEL, Shanghai, China, 416 www.anpel.com.cn/cnw) and filtrated (SCAA-104, $0.22 \mu \mathrm{m}$ pore size; ANPEL, 417 Shanghai, China, http://www.anpel.com.cn/) before liquid chromatography-mass 418 spectrometry (LC-MS) analysis.

\section{High-performance liquid chromatography (HPLC) Conditions}

420 The sample extracts were analyzed using an LC-electrospray ionization (ESI)-MS/MS

421 system (HPLC, Shim-pack UFLC SHIMADZU CBM30A system, 422 www.shimadzu.com.cn/; MS, Applied Biosystems 6500 Q TRAP, 423 www.appliedbiosystems.com.cn/). The analytical conditions were as follows, HPLC: 424 column, Waters ACQUITY UPLC HSS T3 C18 $(1.8 \mu \mathrm{m}, 2.1 \mathrm{~mm} * 100 \mathrm{~mm})$; solvent 425 system, water $(0.04 \%$ acetic acid): acetonitrile (0.04\% acetic acid); gradient program, $42695: 5 \mathrm{~V} / \mathrm{V}$ at $0 \mathrm{~min}, 5: 95 \mathrm{~V} / \mathrm{V}$ at $11.0 \mathrm{~min}, 5: 95 \mathrm{~V} / \mathrm{V}$ at $12.0 \mathrm{~min}, 95: 5 \mathrm{~V} / \mathrm{V}$ at $12.1 \mathrm{~min}$, $42795: 5 \mathrm{~V} / \mathrm{V}$ at $15.0 \mathrm{~min}$; flow rate, $0.40 \mathrm{~mL} / \mathrm{min}$; temperature, $40^{\circ} \mathrm{C}$; injection volume: 2 $428 \mu \mathrm{L}$. The effluent was alternatively connected to an ESI-triple quadrupole-linear ion $429 \operatorname{trap}(\mathrm{Q}$ TRAP)-MS 
431 Linear ion trap (LIT) and triple quadrupole (QQQ) scans were acquired on a triple

432 quadrupole-linear ion trap mass spectrometer (Q TRAP) API 6500 Q TRAP

433 LC/MS/MS System equipped with an ESI Turbo Ion-Spray interface, operating in

434 positive ion mode and controlled by Analyst 1.6.3 software (AB Sciex). The ESI

435 source operation parameters were as follows: ion source, turbo spray; source

436 temperature, $500^{\circ} \mathrm{C}$; ion spray voltage (IS), $5500 \mathrm{~V}$; ion source gas I (GSI), gas II

437 (GSII), and curtain gas (CUR) were set at 55, 60, and $25.0 \mathrm{psi}$, respectively; the

438 collision gas (CAD) was high. Instrument tuning and mass calibration were performed

439 with 10 and $100 \mu \mathrm{mol} / \mathrm{L}$ polypropylene glycol solutions in QQQ and LIT modes,

440 respectively. QQQ scans were acquired as multiple reaction monitoring (MRM)

441 experiments with collision gas (nitrogen) set to 5 psi. DP and CE for individual MRM

442 transitions were done with further DP and CE optimization. A specific set of MRM

443 transitions was monitored for each period according to the metabolites eluted within

444 this period.

445 Qualitative and Quantitative Determination of Metabolites

446 Based on a self-established database (MWDB) and a public metabolite database, 447 primary and secondary mass spectrometry data were subjected to qualitative analyses.

448 For the qualitative analyses of some substances, interference from isotope signals;

449 duplicate signals of $\mathrm{K}^{+}, \mathrm{Na}^{+}$, and $\mathrm{NH}_{4}{ }^{+}$ions; and duplicate signals of fragment ions 450 derived from other relatively large molecules were excluded (Fraga et al. 2010; Yang, 451 et al. 2019).

\section{Statistical Analysis}

453 Principal component analysis (PCA), two-way analysis of variance (ANOVA), 
454 ANOVA-simultaneous component analysis (ASCA), and heat maps were generated 455 in $\mathrm{R}$ (base package) version 3.5.0. The data were normalized prior to analysis. Two 456 screening criteria for significant differential metabolites were applied, including a fold 457 change of $\geq 2$ or of $\leq 0.5$ and a $P$-value $<0.05$ (Student's $t$-test). 
Additional file 1: Figure S1. Rice pedigree analysis based on information from Ricedata (http://www.ricedata.cn). Figure S2. Major patterns associated with time and cultivars (developmental stage). Figure S3. ANOVA-simultaneous component analysis. Figure S4. Relative changes in essential amino acids in the rice grains during the development. Blue, red, green, and purple represent MXZ, MSZ, HHZ, and QXZ respectively. Figure S5. Relative changes in vitamins in the rice grains during development. Blue, red, green, and purple represent the MXZ, MSZ, HHZ, and QXZ samples, respectively. Figure S6. Classes of metabolites significantly reduced (a) and increased (b) in the four cultivars during the postharvesting process (from 30 to $40 \mathrm{DAF}$ ). Figure S7. Percent of chlorpyrifos remaining in the sample (40 DAF) after the post-harvesting process. Figure S8. Classes of metabolites significantly lower (a) and higher (b) in the rice samples (40 DAF) in MSZ, HHZ, and QXZ compared with MXZ. Table S1. A list of the 623 metabolites detected in this study. Table S2.

471 Time List of well-modeled metabolites. Table S3. Cultivar List of well-modeled metabolites. Table S4. Interaction List of well-modeled metabolites. Table S5. Carbohydrate with

473 significant changes at 8 DAF. Table S6. Carbohydrate with significant changes at 30 DAF.

474 Table S7. Amino acid and derivatives with significant changes at 8 DAF. Table S8. Amino 475 acid and derivatives with significant changes at 15 DAF. Table S9. Amino acid and 476 derivatives with significant changes at $30 \mathrm{DAF}$.

\section{Abbreviations}

MXZ: MeiXiangZhan2; MSZ: MeiSiZhan; HHZ: HuangHuaZhan; QXZ: QiXinZhan;

479 DAF: days after flowering; PCA: principal component analysis; ANOVA: two-way 480 analysis of variance; ASCA: ANOVA-simultaneous component analysis; UPDG: 481 uridine diphosphate glucose; G-6-P: Glucose-6-phosphate; F-6-P: fructose-6- 
482 phosphate; G-1-P: Glucose-1-phosphate; LysoPEs: lysophosphatidylethanolamine;

483 MAGs: monoacylglycerols; LysoPCs: lysophosphatidylcholine.

484 Acknowledgement

485 We thank WuHan Metware and Guangzhou Gene Denovo Biotechnology Co. Ltd., 486 for assisting in the metabolite and bioinformatics analyses.

$487 \quad$ Funding

488 This work was financially supported by the Natural Science Foundation of 489 Guangdong Province (2018A030313465 and 2015B020231001), the National High490 tech R\&D Program of China (863 Program, 2014AA10A604-19), the National 491 Natural Science Foundation of China (31801448), and the

492 Special fund for scientific innovation strategy-

493 construction of high level Academy of Agriculture Science.

494 Ethics Approval and Consent to Participate

495 Not applicable.

496 Consent for Publication

497 Not applicable.

498 Competing Interests

499 The authors declare no potential competing interests.

500 Author details

501 Yi-Bo Chen and Zhidong Wang contributed equally to this work. 
502 Affiliations: 1Guangdong Academy of Agricultural Sciences, Rice Research Institute, 503 Guangzhou, 510640, China. 2Guangdong Key Laboratory of New Technology 504 in Rice Breeding, Guangzhou, 510640, China.

505

506 
Aguilar C, Gavino G, Baragano M, Hevia P, Gavino C (2007) Correlation of tocopherol, tocotrienol, $\gamma$-oryzanol and total polyphenol content in rice bran with different antioxidant capacity assays. Food Chem 102:1228-1232. doi:10.1016/j.foodchem.2006.07.012.

Amir R, Galili G, Cohen H (2018) The metabolic roles of free amino acids during seed development. Plant Sci 275:11-18. doi:10.1016/j.plantsci.2018.06.011.

Chen W, Wang W, Peng M, Gong L, Gao Y, Wan J, Wang S, Shi L, Zhou B, Li Z (2016) Comparative and parallel genome-wide association studies for metabolic and agronomic traits in cereals. Nat Commun 7:12767. doi:10.1038/ncomms127671.

Chishti Z, Hussain S, Arshad R, Khalid A, Arshad M (2013) Microbial degradation of chlorpyrifos in liquid media and soil. J Environ Manage 114:372-380. doi:10.1016/j.jenvman.2012.10.032.

Corrêa P, Da S, Jaren C, Júnior A, Arana I (2007) Physical and mechanical properties in rice processing. J Food Eng 79:137-142. doi:10.1016/j.jfoodeng.2006.01.037.

Deng Y, Gong Y, Wang T (2013) Use of proteomics to understand seed development in rice. Proteomics 13: 1784-1800. doi:10.1002/pmic.201200389.

Fraga G, Clowers H, Moore J, Zink M (2010) Signature-discovery approach for sample matching of a nerve-agent precursor using liquid chromatography-mass spectrometry, XCMS, and chemometrics. Anal Chem 82:4165-4173. doi:10.1021/ac1003568.

Hori K (2018) Genetic dissection and breeding for grain appearance quality in rice. In Rice Genomics, Genetics and Breeding, Springer, p 435.

Hu C, Tohge T, Chan A, Song Y, Rao J, Cui B, Lin H, Wang L, Fernie R, Zhang D (2016) Identification of conserved and diverse metabolic shifts during rice grain development. Sci Rep 6: 20942. doi:10.1038/srep20942.

Jang A, Park W, Kwon E, Song S, Park B, Jeon H, Sohn H, Koo J, Kang C (2017) Quinic acid inhibits vascular inflammation in TNF- $\alpha$-stimulated vascular smooth muscle cells. Biomed Pharmacother 96:563-571. doi:10.1016/j.biopha.2017.10.021.

Kara V, Aldemir N, Ozcicek F, Mammadov R (2019) Protective Effect of Taxifolin on Cisplatin-Induced Nephrotoxicity in Rats. Anal Quant Cytopatho 41:47-54.

Liu L, Waters L, Rose J, Bao J, King J (2013) Phospholipids in rice: significance in grain quality and health benefits: a review. Food Chem 139:1133-1145. doi: 10.1016/j.foodchem.2012.12.046.

Sreenivasulu N, Butardo M, Misra G, Cuevas P, Anacleto R, Kavi B (2015) Designing climate-resilient rice with ideal grain quality suited for high-temperature stress. J Exp Bot 66:1737-1748. doi: 10.1093/jxb/eru544.

Sulpice R (2019) Closing the yield gap: can metabolomics be of help? J Exp Bot doi:10.1093/jxb/erz322. doi: 10.1093/jxb/erz322.

Taiwo A, Bart A (2016) Factors Responsible for Post-Harvest Losses and Their Effects on Rice Producing Farmers: A Case Study of Afife and Aveyime Rice Projects in the Volta Region Of Ghana.

Tian Z, Qian Q, Liu Q, Yan M, Liu X, Yan C, Liu G, Gao Z, Tang S, Zeng D (2009) Allelic 

diversities in rice starch biosynthesis lead to a diverse array of rice eating and cooking qualities. PNAS 106:21760-21765. doi: 10.1073/pnas.0912396106.

Wang C, Xu H, Zhu Y, Liu Q, Cai L (2013) OsbZIP58, a basic leucine zipper transcription factor, regulates starch biosynthesis in rice endosperm. J Exp Bot 64:3453-3466. doi: $10.1093 / \mathrm{j} x \mathrm{~b} / \mathrm{ert} 187$.

Wang X, Zhou W, Lu Z, Ouyang Y, O, S, Yao J (2015) A lipid transfer protein, OsLTPL36, is essential for seed development and seed quality in rice. Plant Sci 239:200-208. doi: 10.1016/j.plantsci.2015.07.016.

Xu J, Xu Z, Zheng W (2017) A review of the antiviral role of green tea catechins. Molecules 22:1337. doi: $10.3390 /$ molecules 22081337.

Yan S, Huang W, Gao J, Fu H, Liu J (2018) Comparative metabolomic analysis of seed metabolites associated with seed storability in rice (Oryza sativa L.) during natural aging. Plant Physiol Bioch 127:590-598. doi: 10.1016/j.plaphy.2018.04.020.

Yang M, Yang J, Su L, Sun K, Li D, Liu Y, Wang H, Chen Z, Guo T (2019) Metabolic profile analysis and identification of key metabolites during rice seed germination under lowtemperature stress. Plant Sci 289:110282. doi: 10.1016/j.plantsci.2019.110282.

Yang Y, Guo M, Sun S, Zou Y, Yin S, Liu Y, Tang S, Gu M, Yang Z, Yan C (2019) Natural variation of OsGluA2 is involved in grain protein content regulation in rice. Nat Commun 10:1949. doi: 10.1038/s41467-019-09919-y.

Zarei I, Brown G, Nealon J, Ryan P (2017) Rice bran metabolome contains amino acids, vitamins \& cofactors, and phytochemicals with medicinal and nutritional properties. Rice 10:24. doi: 10.1186/s12284-017-0157-2.

Zhao S, Li F, Zhang Q, Zhang C, Yang Q, Pan X, Ren Y, Lu J, Gu H, Liu Q (2018) GS9 acts as a transcriptional activator to regulate rice grain shape and appearance quality. Nat Commun 9:1240. doi: 10.1038/s41467-018-03616-y.

Zhou M, Ibrahim K (2009) Tricin - a potential multifunctional nutraceutical. Phytochem Rev 9: 413-424. doi: 10.1007/s11101-009-9161-5.

Zhu G, Wang S, Huang Z, Zhang S, Liao Q, Zhang C, Lin T, Qin M, Peng M, Yang C (2018) Rewiring of the fruit metabolome in tomato breeding. Cell 172:249-261. doi: 10.1016/j.cell.2017.12.019.

Zhu Q, Wang B, Tan J, Liu T, Li L, Liu G, Wersch S, Tian L, Hoy R, Li X (2019) Plant synthetic metabolic engineering for enhancing crop nutritional quality. Plant Commun 100017. doi:10.1016/j.xplc.2019.100017. 
Fig. 1. Chen et al.

(a)

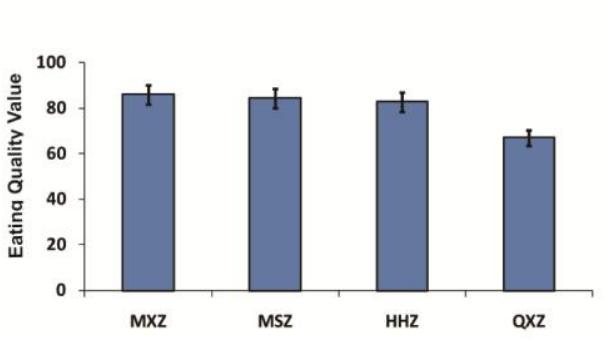

(b)

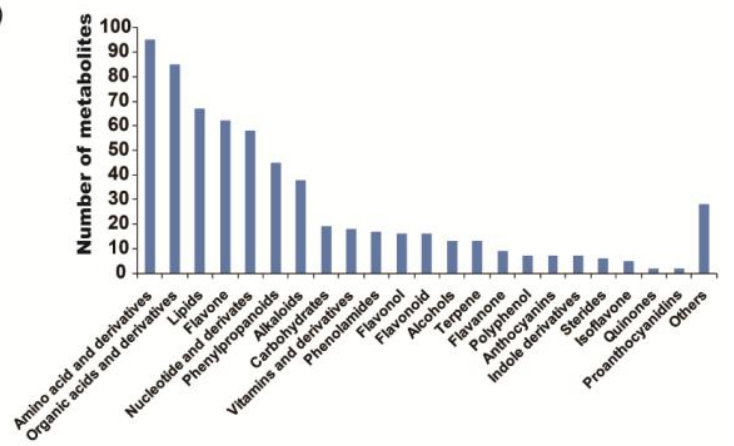

(c)

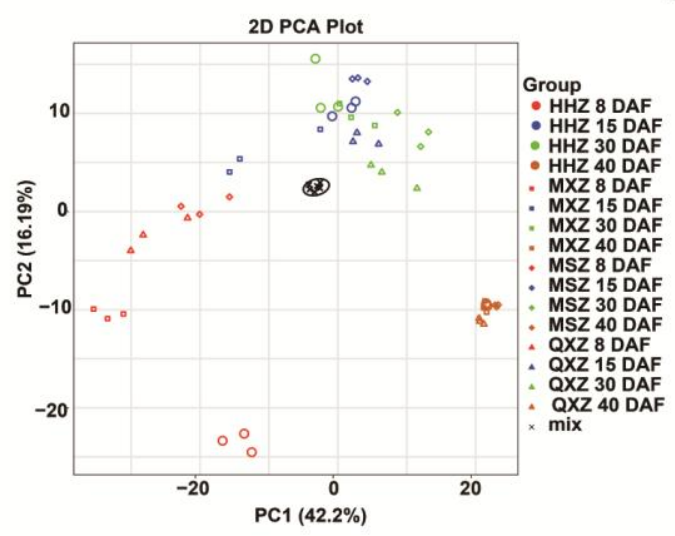

(d)

Two-way ANOVA (within subject)

586 Fig. 1. (a) Eating quality values of MXZ, MSZ, HHZ, and QXZ; (b) metabolite classes and 587 numbers detected in the samples; (c) principal component analysis (PCA) of the metabolomes

588 during grain development and the post-harvesting process. Red, blue, green, and orange 589 represent samples at $8,15,30$, and $40 \mathrm{DAF}$, respectively. The circle, square, rhombus, and 590 triangle denote the grain metabolomes of HHZ, MXZ, MSZ, and QXZ, respectively; (d) Venn 591 diagram summary of the results from the two-way ANOVA. 
Fig. 2. Chen et al.

(a)

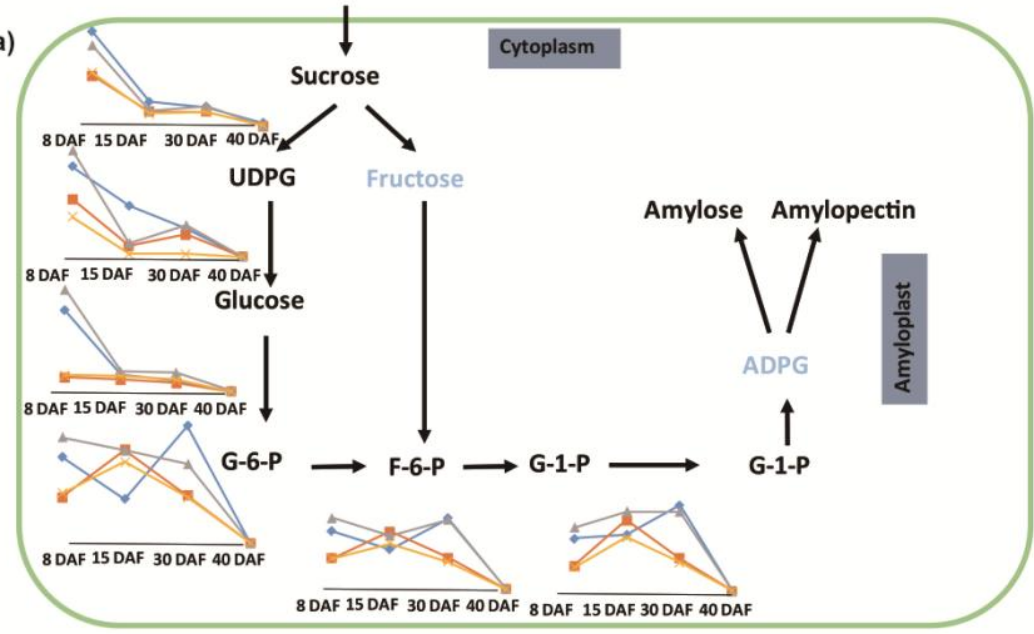

(b)

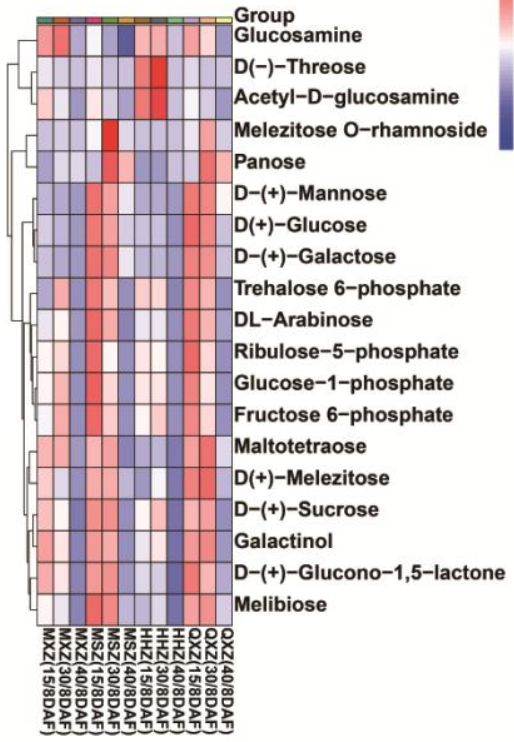

(c)

$\rightarrow-\mathrm{MXZ} \rightarrow-\mathrm{MSZ} \rightarrow-\mathrm{HHZ} \rightarrow-\mathrm{QXZ}$

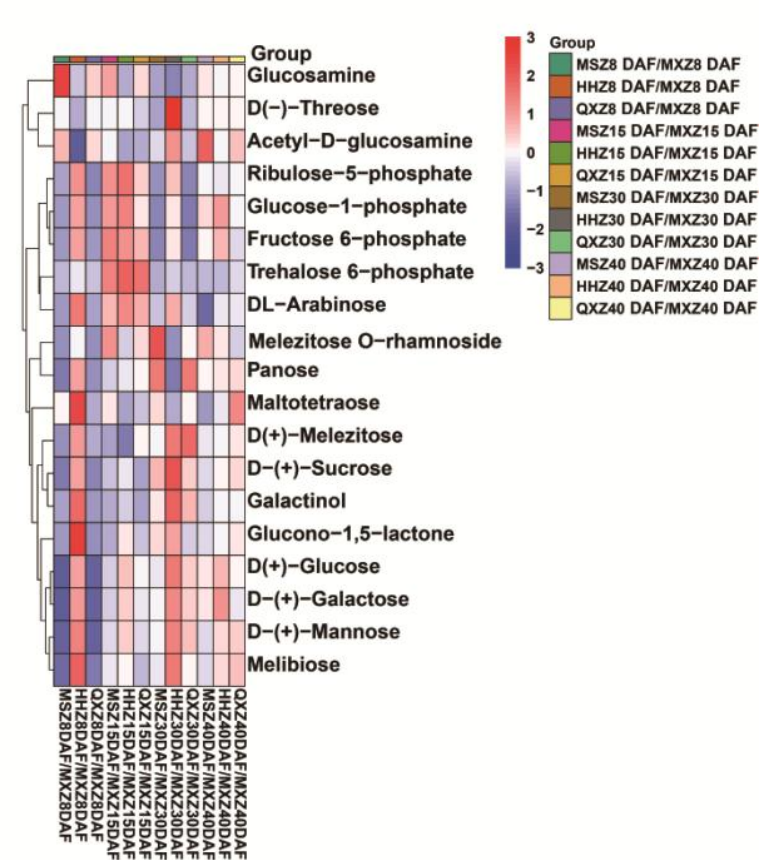

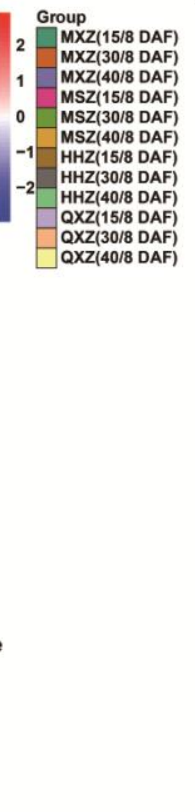

593 Fig. 2. (a) Changes in metabolites mapped to the starch biosynthesis pathway in the four rice

594 cultivars at 8, 15, 30, and 40 DAF. Abbreviations: UPDG, uridine diphosphate glucose; G-6-P,

595 Glucose-6-phosphate; F-6-P, fructose-6-phosphate; G-1-P, Glucose-1-phosphate; (b) and (c)

596 Heatmap of carbohydrate metabolite changes in the rice grains at $8,15,30$, and 40 DAF

597 among the four cultivars (b) or compared with MXZ (c). Ratios of fold changes are given by

598 shades of red or blue according to the scale bar. Data represent the mean values of three 
599 biological replicates for each cultivar and time point. For full metabolite names, refer to Table

600 S1.

601

Fig. 3. Chen et al.

(a)

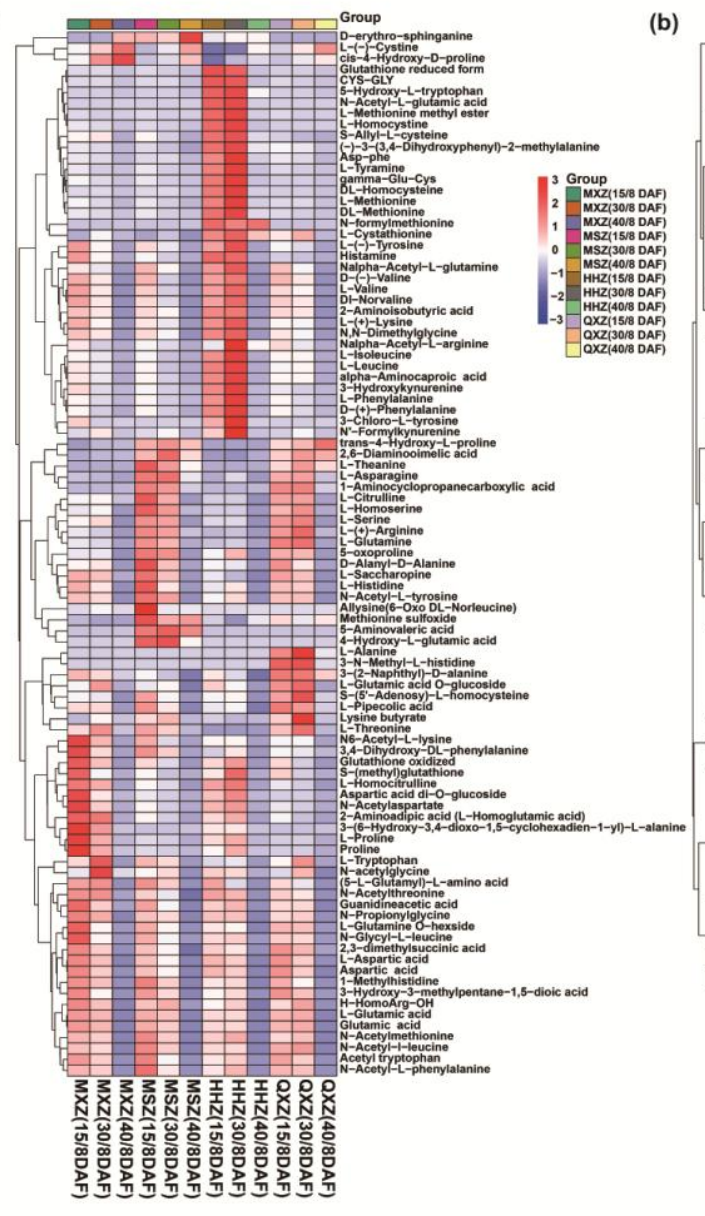

(b)

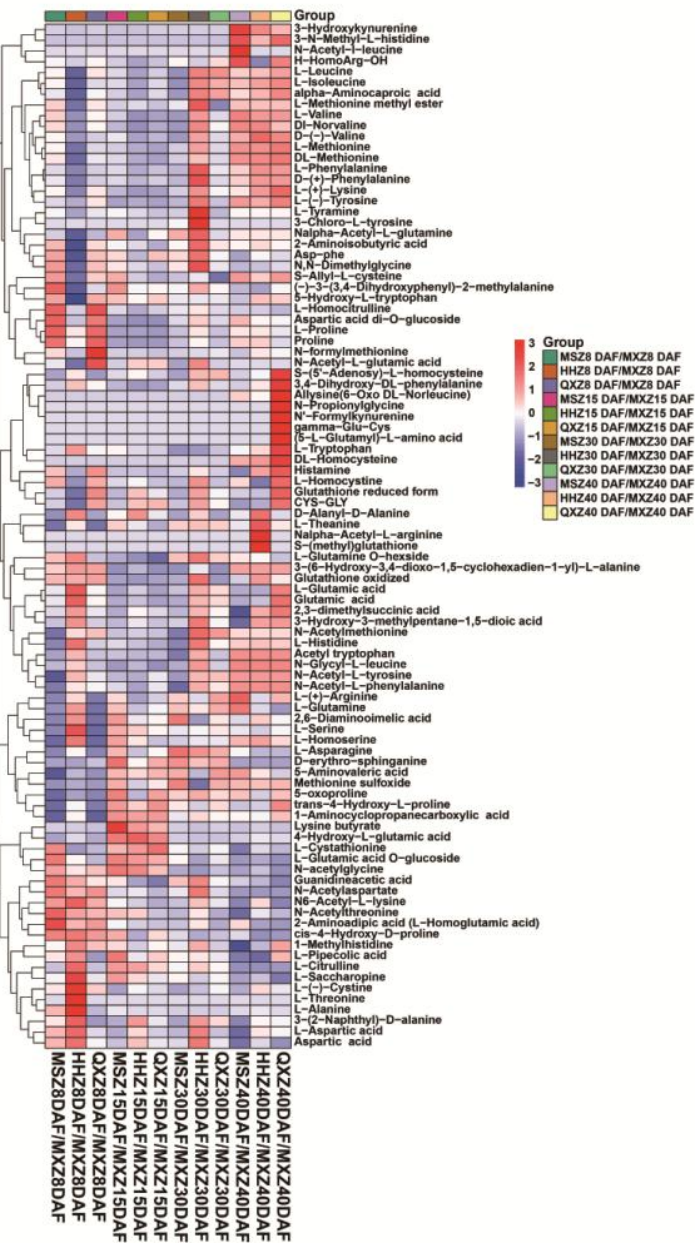

602

603 Fig. 3. Heatmap of the changes in amino acids and derivatives in the rice grains at 8, 15, 30, 604 and 40 DAF among the four cultivars (b) or compared with MXZ (c). Ratios of fold changes 605 are given by shades of red or blue according to the scale bar. Data represent the mean values 606 of three biological replicates for each cultivar and time point. For full metabolite names, refer 607 to Table S1. 

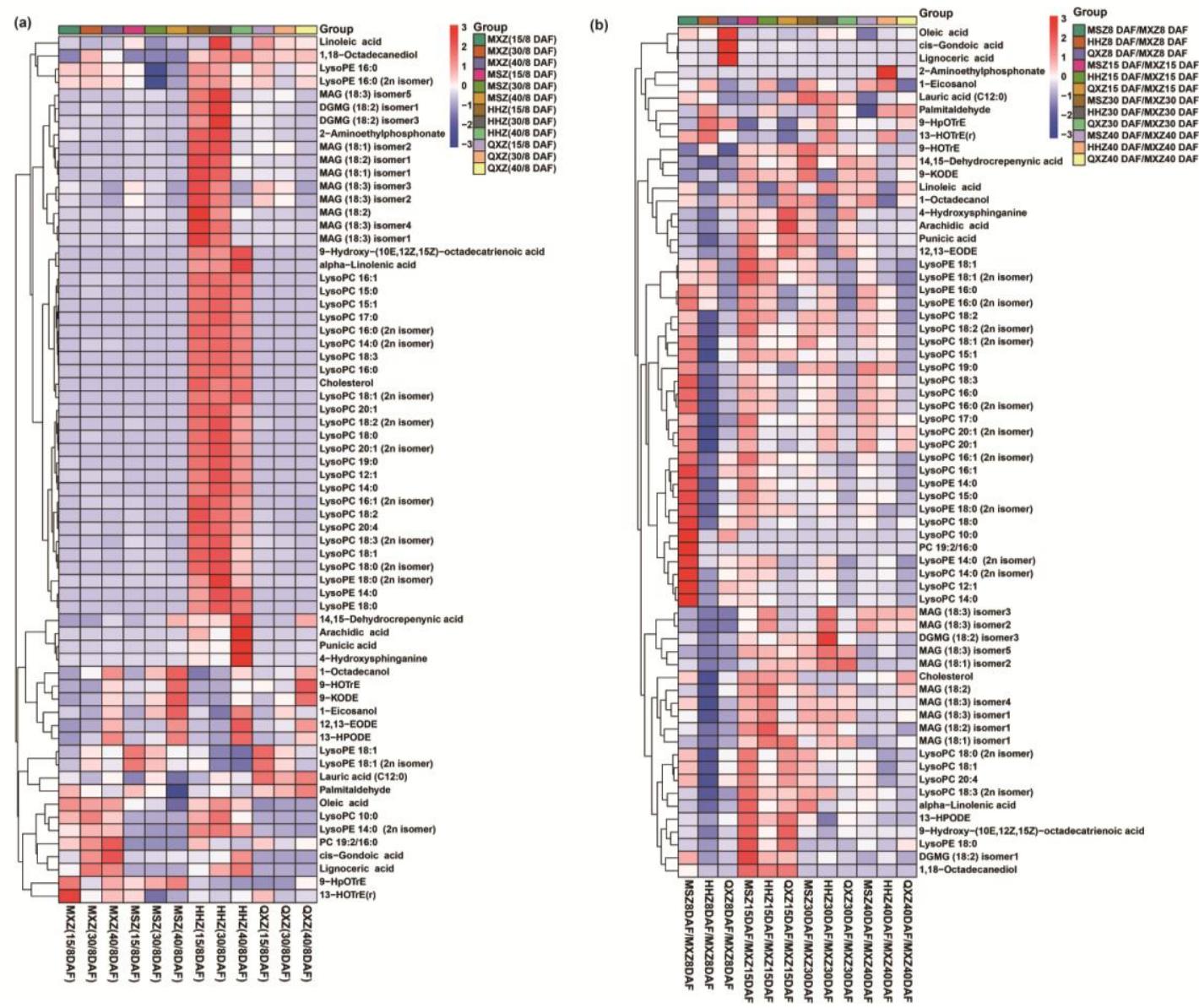

610 Fig. 4. Heatmap of lipid metabolite changes in the rice grains at 8, 15, 30, and $40 \mathrm{DAF}$ among

611 the four cultivars (b) or compared with MXZ (c). Ratios of fold changes are given by shades 612 of red or blue according to the scale bar. Data represent mean values of three biological 613 replicates for each cultivar and time point. For full metabolite names, refer to Table S1. 
(a)

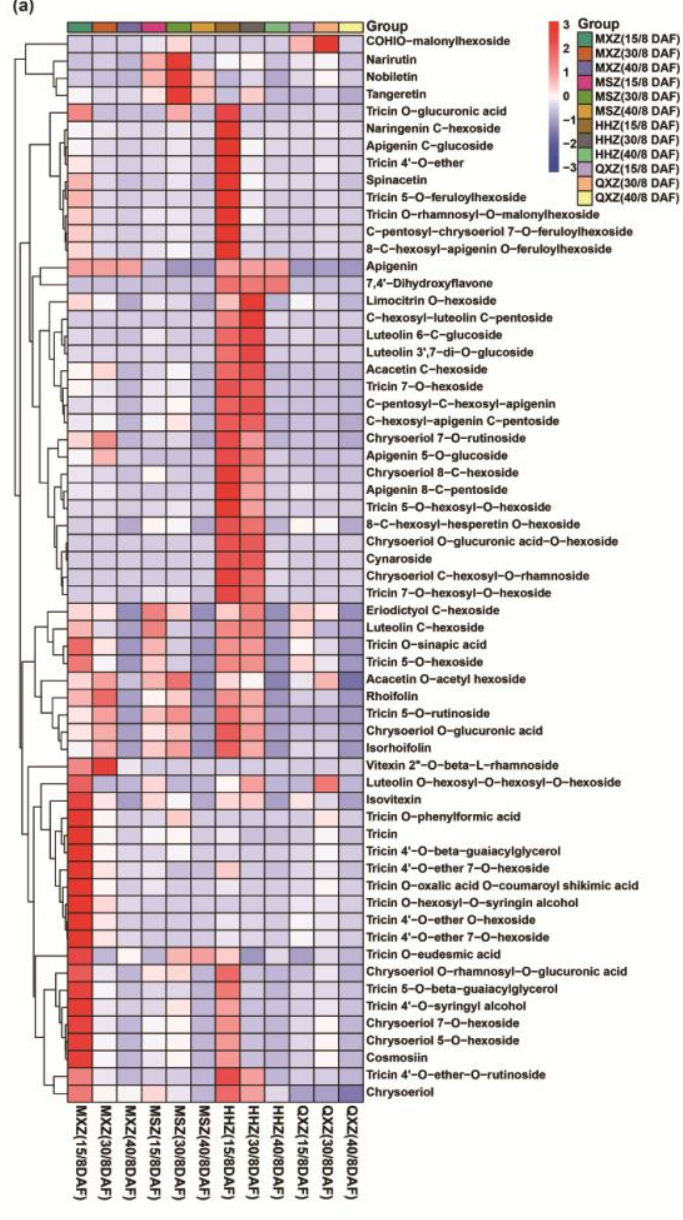

(b)

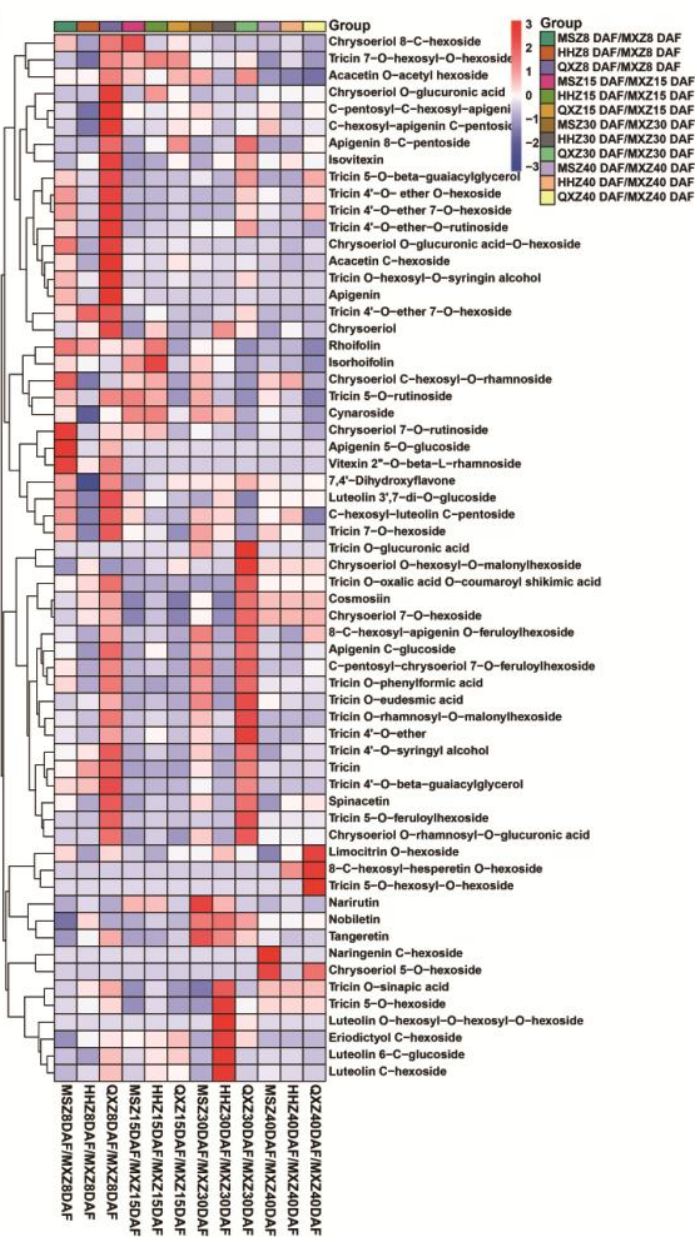

615 Fig. 5. Heatmap of flavones metabolite changes in the rice grains at 8, 15, 30, and 40 DAF

616 among the four cultivars (b) or compared with MXZ (c). Ratios of fold changes are given by

617 shades of red or blue according to the scale bar. Data represent mean values of three

618 biological replicates for each cultivar and time point. For full metabolite names, refer to Table

619 S1. 
Fig. 6. Chen et al.

(a)

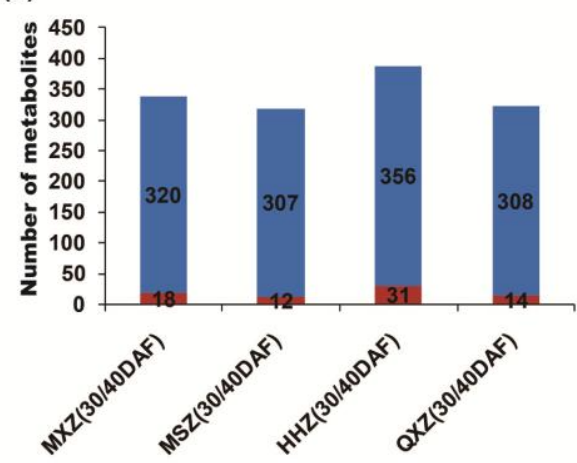

(c)

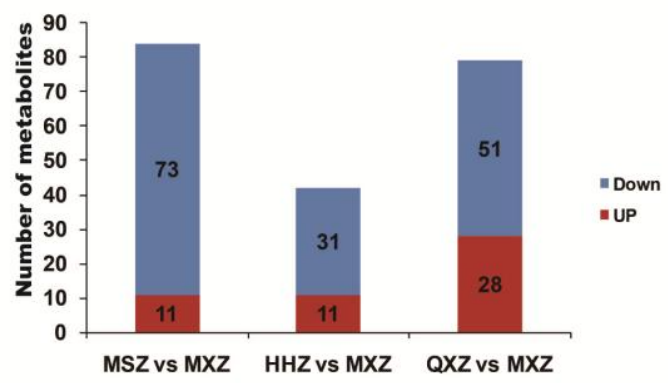

(b)

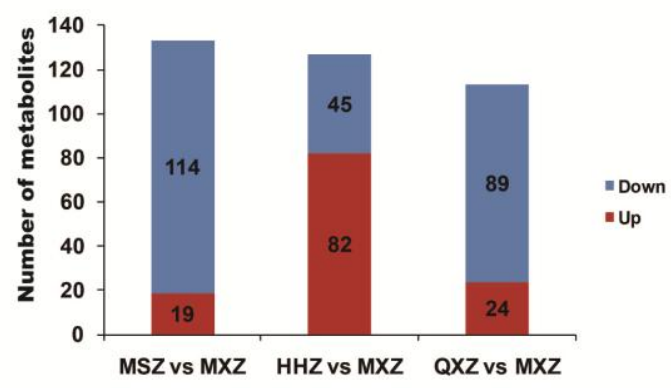

(d)

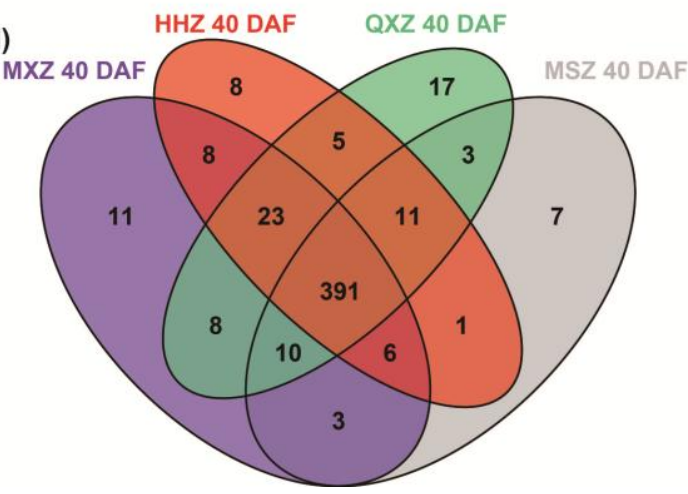

621 Fig. 6. (a) Number of metabolites with differential changes $\geq 2$-fold and $<0.5$-fold $(P<0.05)$

622 in the four cultivars during the post-harvesting process (from 30 to 40 DAF). Number of

623 metabolites with differential changes in MSZ, HHZ, and QXZ compared with MXZ at 30

624 DAF (b) and 40 DAF (white rice) (c). (d) Venn diagram of significantly changed metabolites

625 in the four cultivars at $40 \mathrm{DAF}$ (white rice). 
628

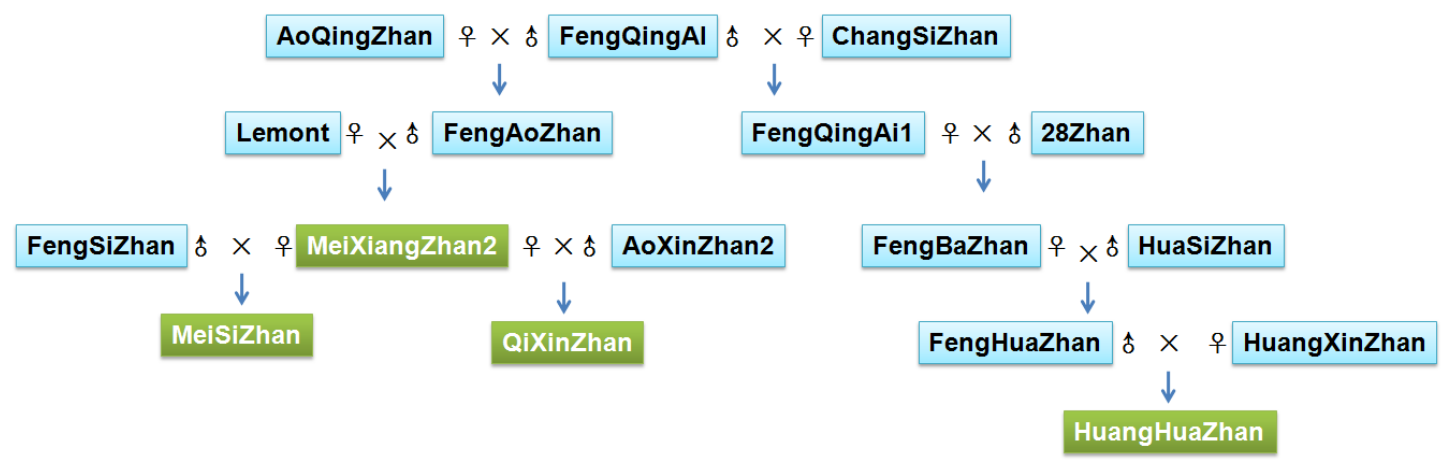

629

Fig. S1. Rice pedigree analysis based on information from Ricedata (http://www.ricedata.cn).

(a)

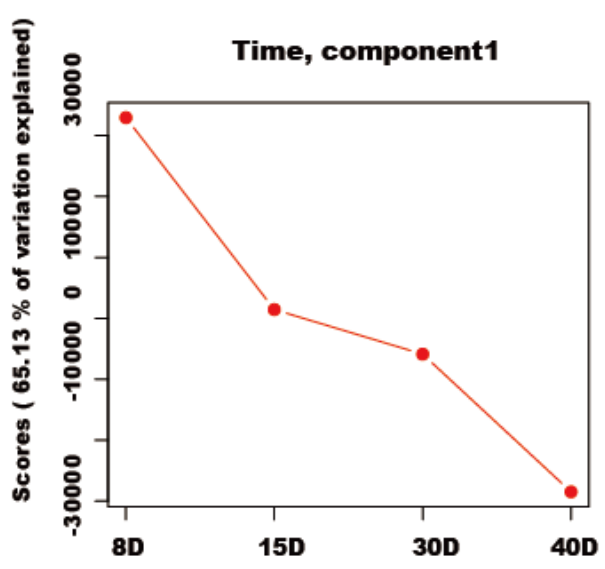

(b)

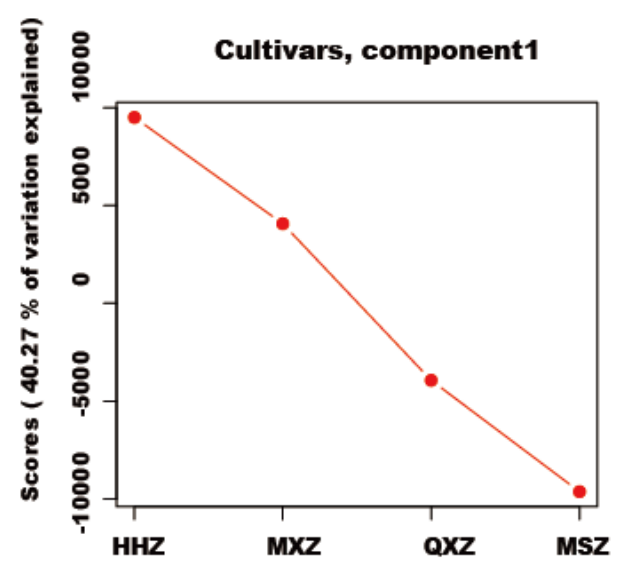

631

632 Fig. S2. Major patterns associated with time and cultivars (developmental stage).

633 

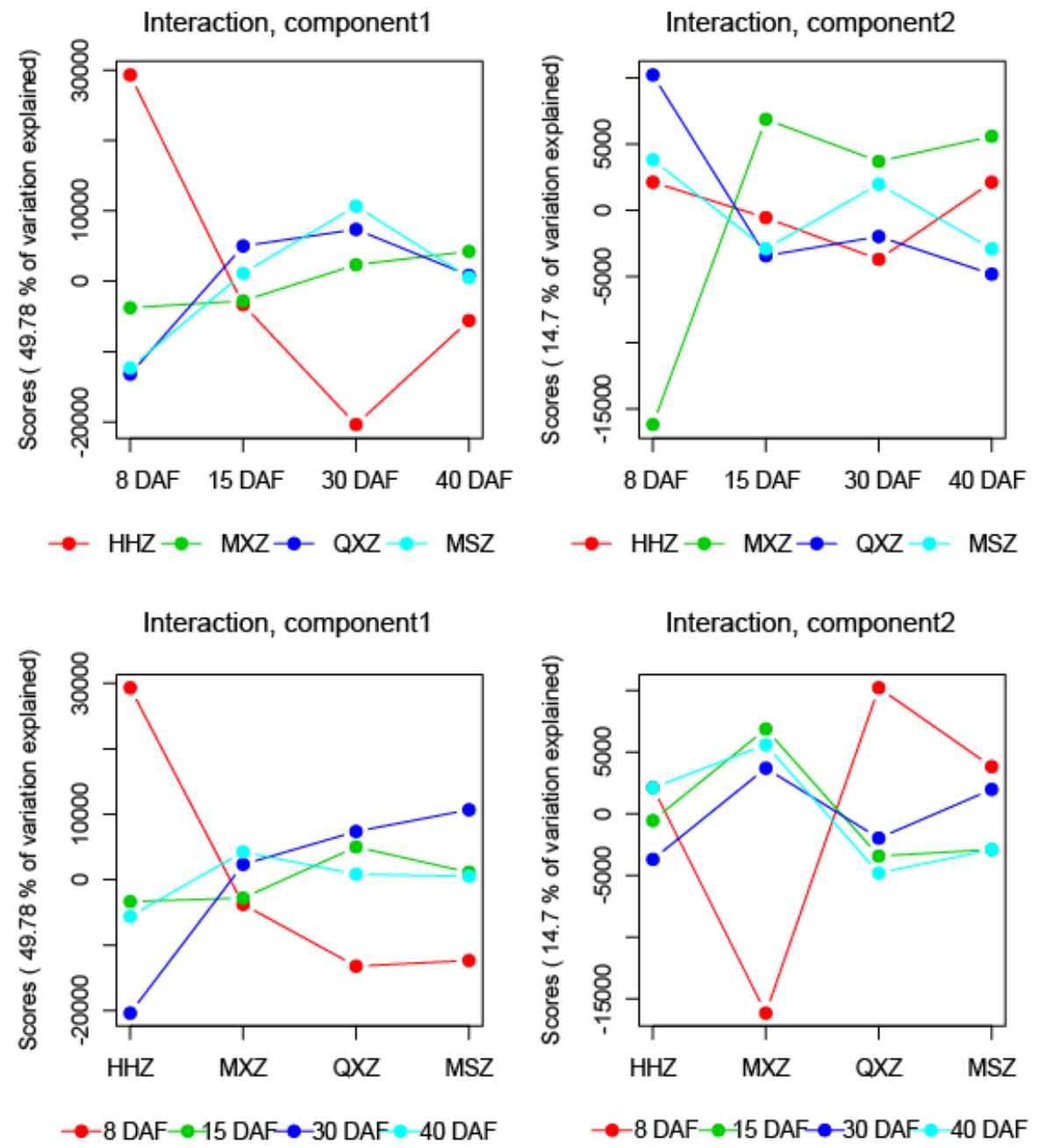

634 -8 DAF-15 DAF- -30 DAF $-40 \mathrm{DAF}$

635 Fig. S3. ANOVA-simultaneous component analysis.

636 

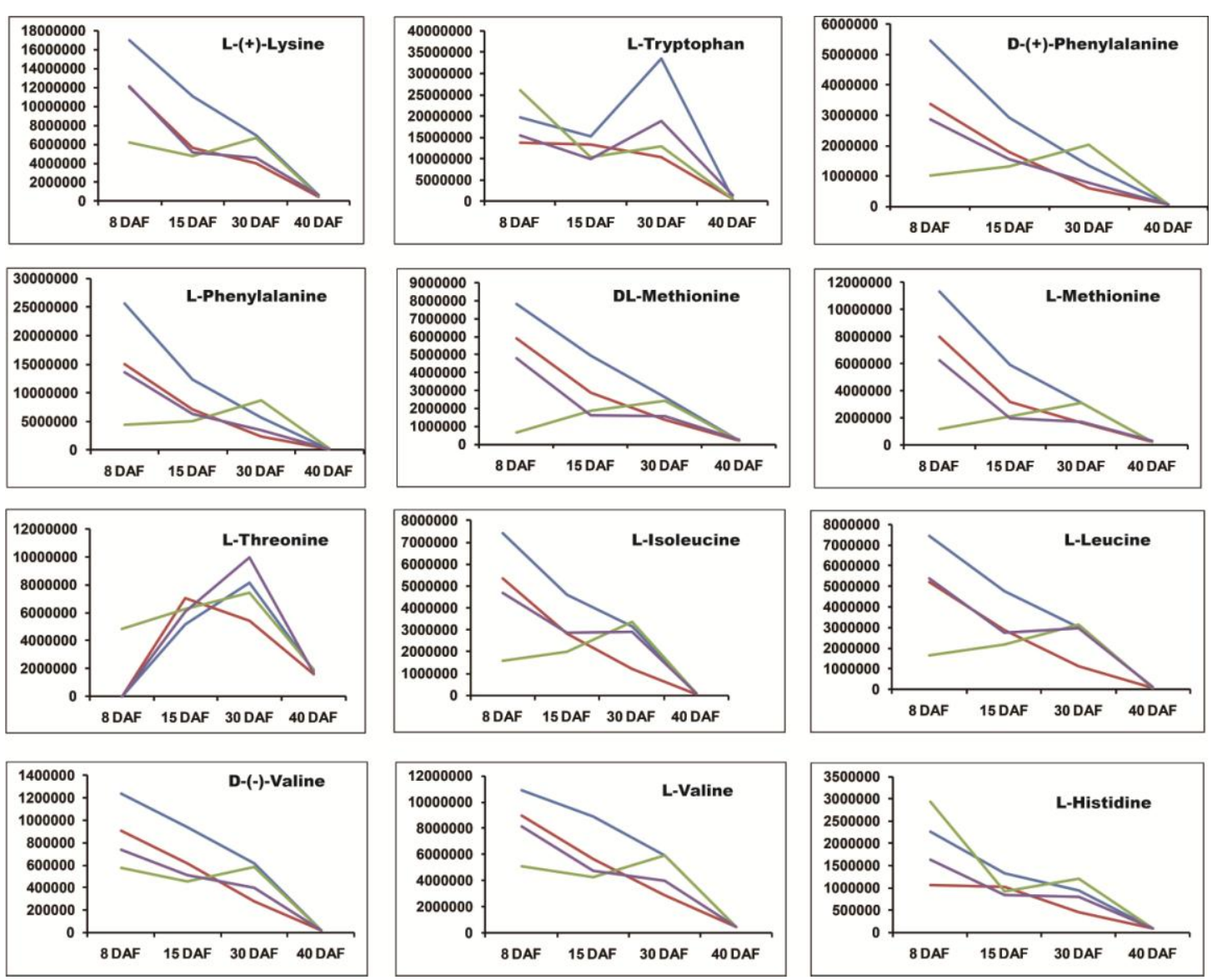

638 Fig. S4. Relative changes in essential amino acids in the rice grains during the development. 639 Blue, red, green, and purple represent MXZ, MSZ, HHZ, and QXZ respectively.
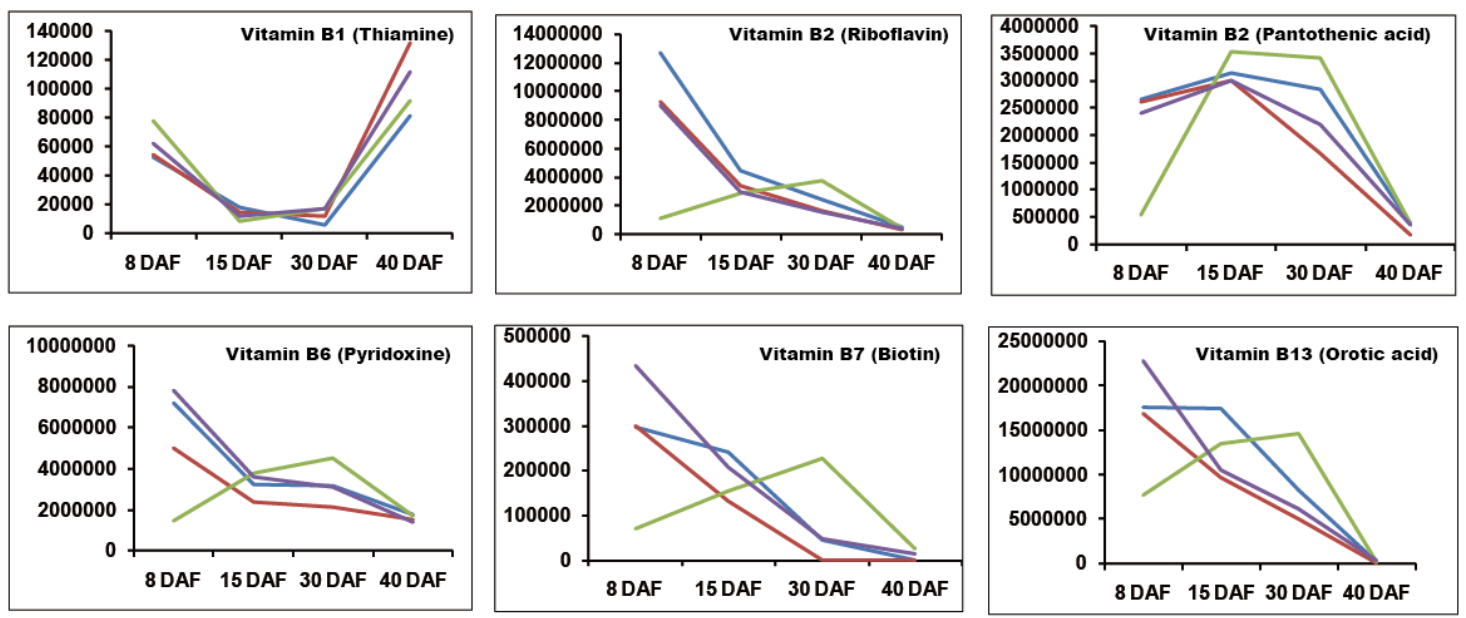

641 Fig. S5. Relative changes in vitamins in the rice grains during development. Blue, red, green, 642 and purple represent the MXZ, MSZ, HHZ, and QXZ samples, respectively. 
(a)

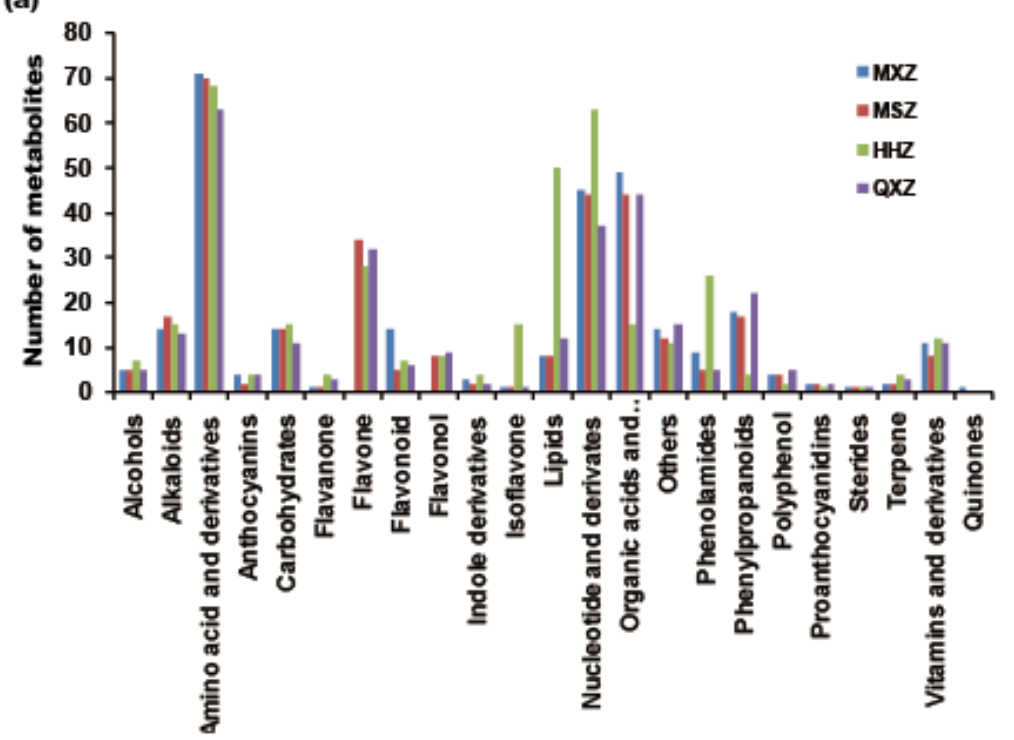

(b)

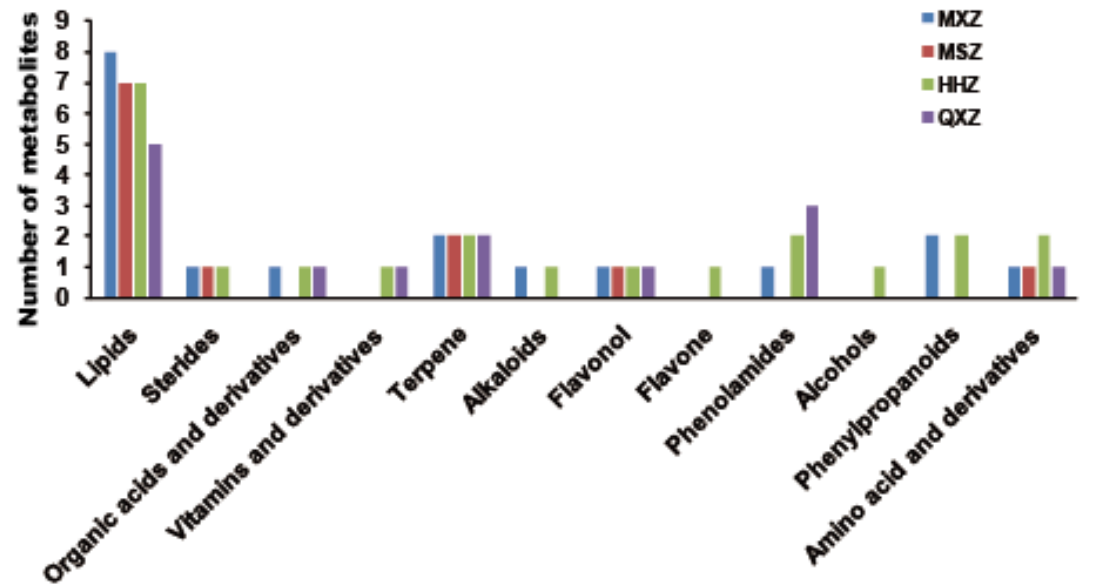

644 Fig. S6. Classes of metabolites significantly reduced (a) and increased (b) in the four 645 cultivars during the post-harvesting process (from 30 to $40 \mathrm{DAF}$ ).

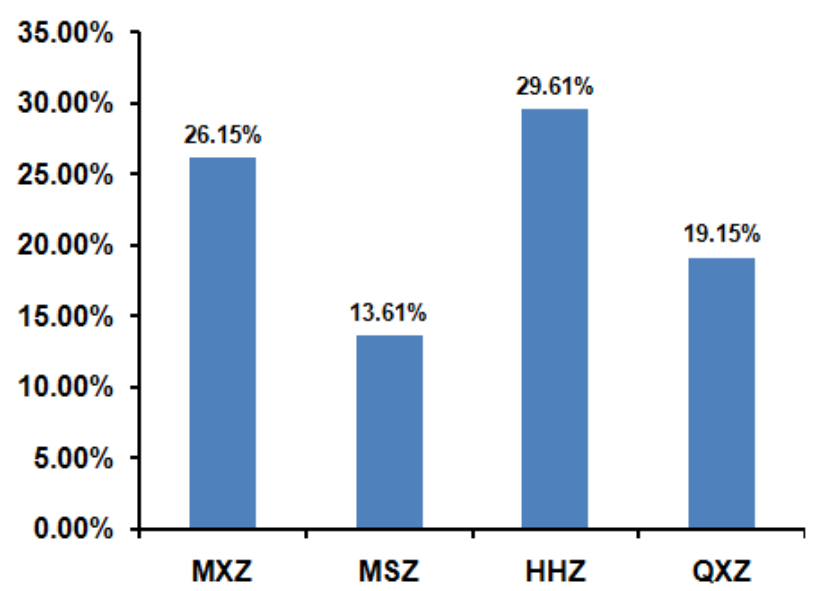

647 Fig. S7. Percent of chlorpyrifos remaining in the sample (40 DAF) after the post-harvesting 648 process. 


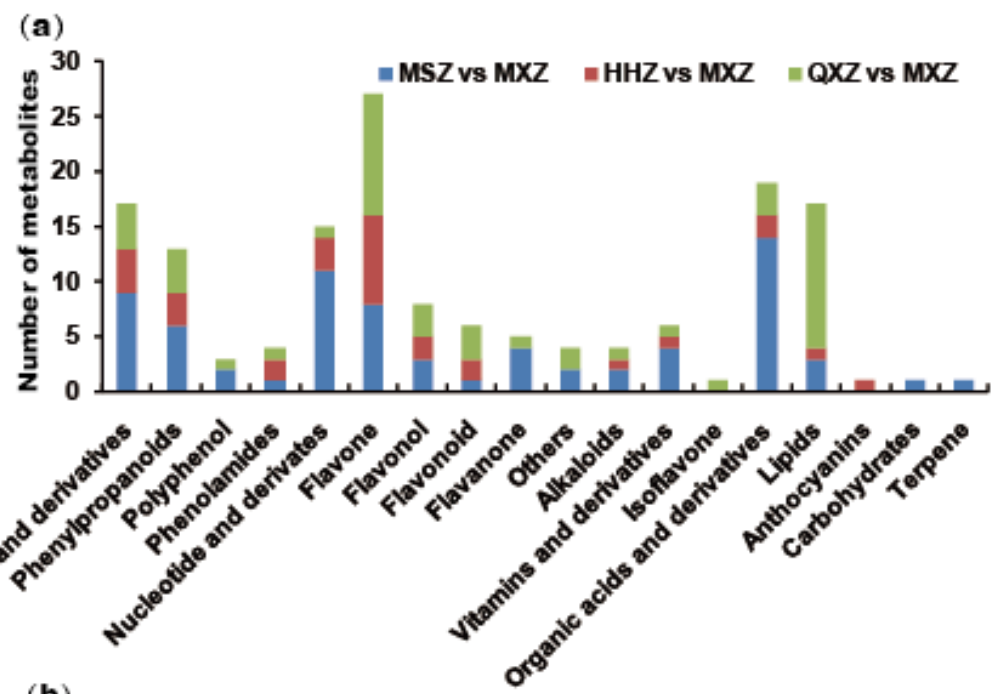

(b)

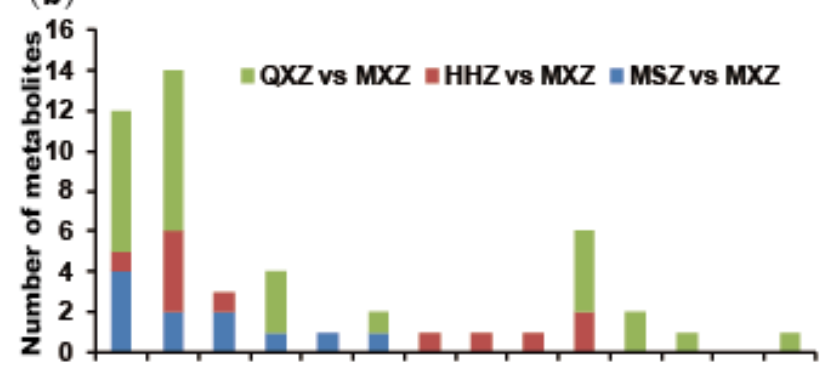

650 Fig. S8. Classes of metabolites significantly lower (a) and higher (b) in the rice samples 651 (40 DAF) in MSZ, HHZ, and QXZ compared with MXZ.

652 


\section{Figures}

(a)

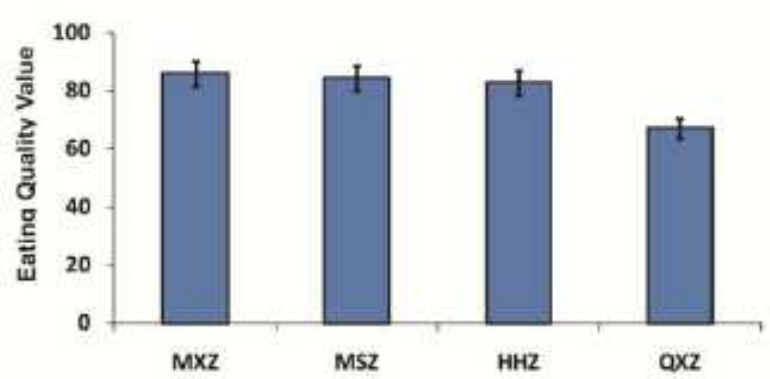

(c)

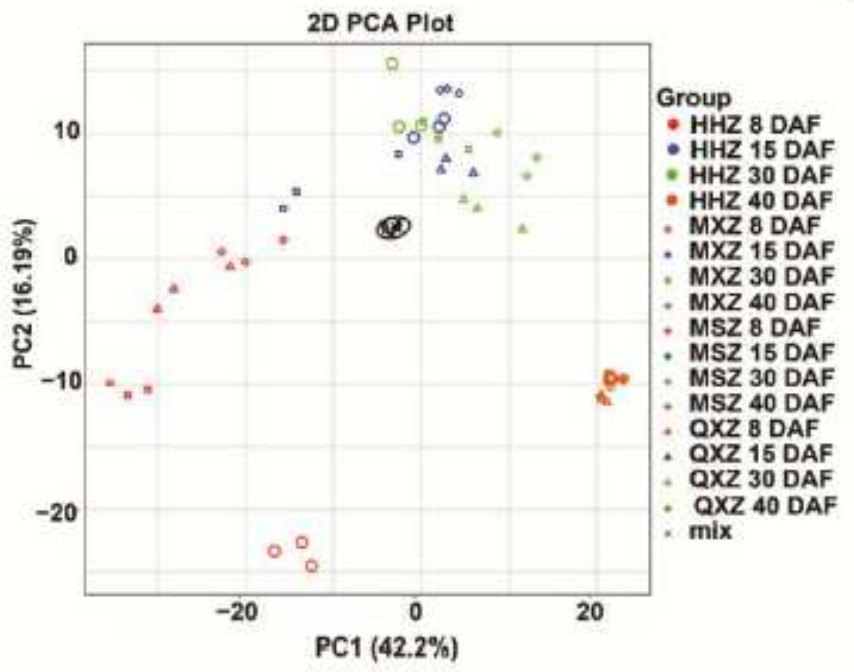

(b)

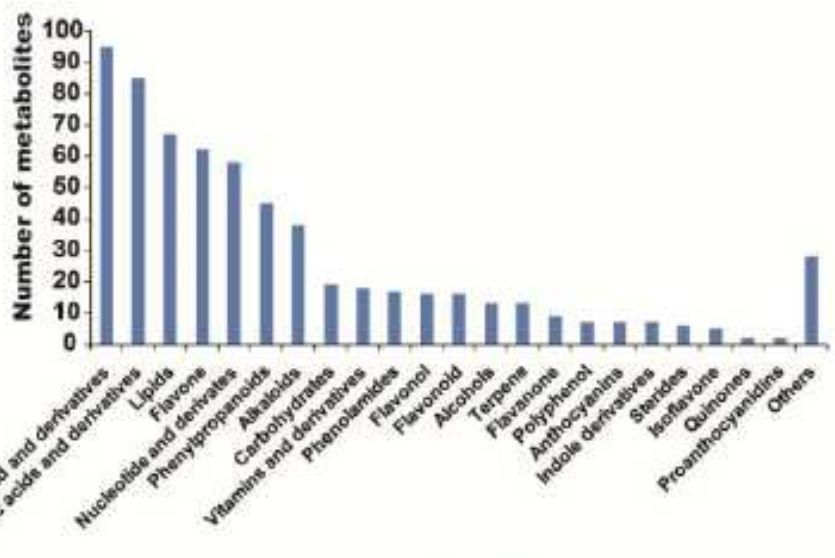

(d)
Two-way ANOVA (within subject)

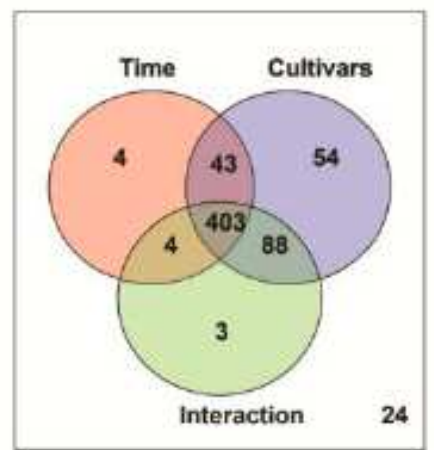

\section{Figure 1}

(a) Eating quality values of MXZ, MSZ, HHZ, and QXZ; (b) metabolite classes and numbers detected in the samples; (c) principal component analysis (PCA) of the metabolomes during grain development and the post-harvesting process. Red, blue, green, and orange represent samples at $8,15,30$, and $40 \mathrm{DAF}$, respectively. The circle, square, rhombus, and triangle denote the grain metabolomes of $\mathrm{HHZ}, \mathrm{MXZ}, \mathrm{MSZ}$, and QXZ, respectively; (d) Venn diagram summary of the results from the two-way ANOVA. 
(a)

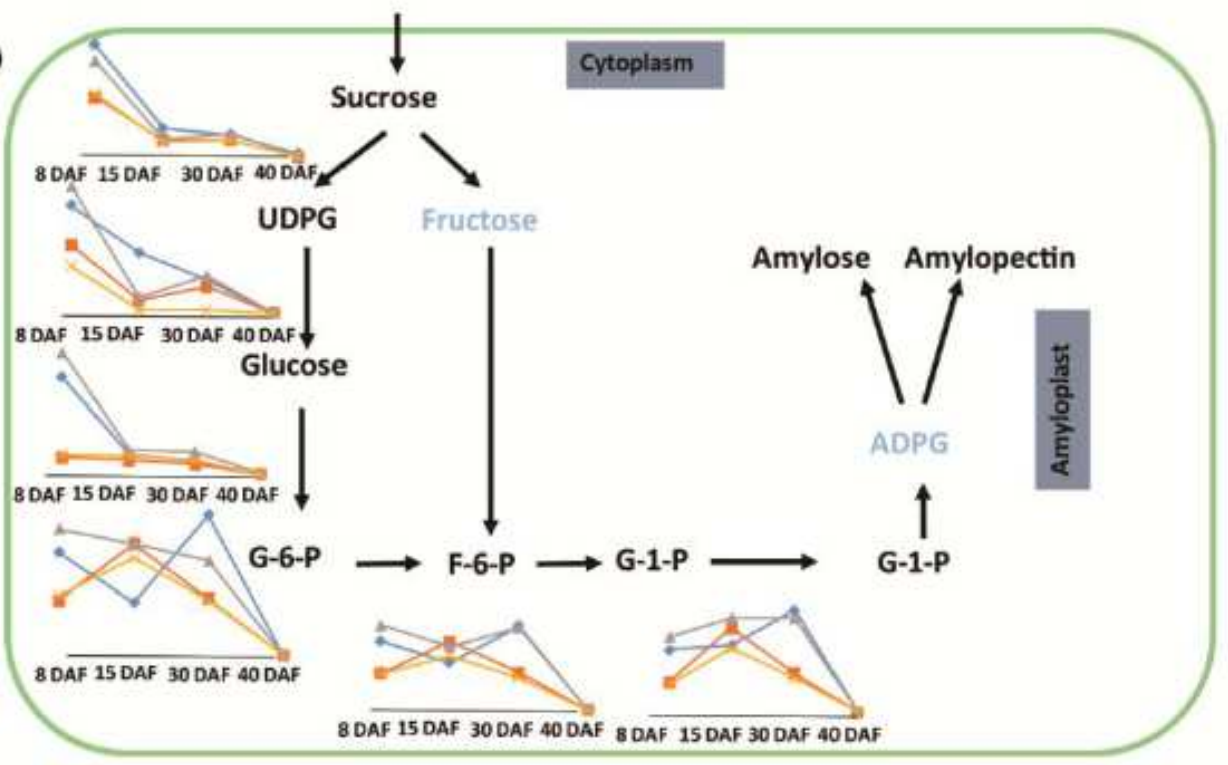

(b)

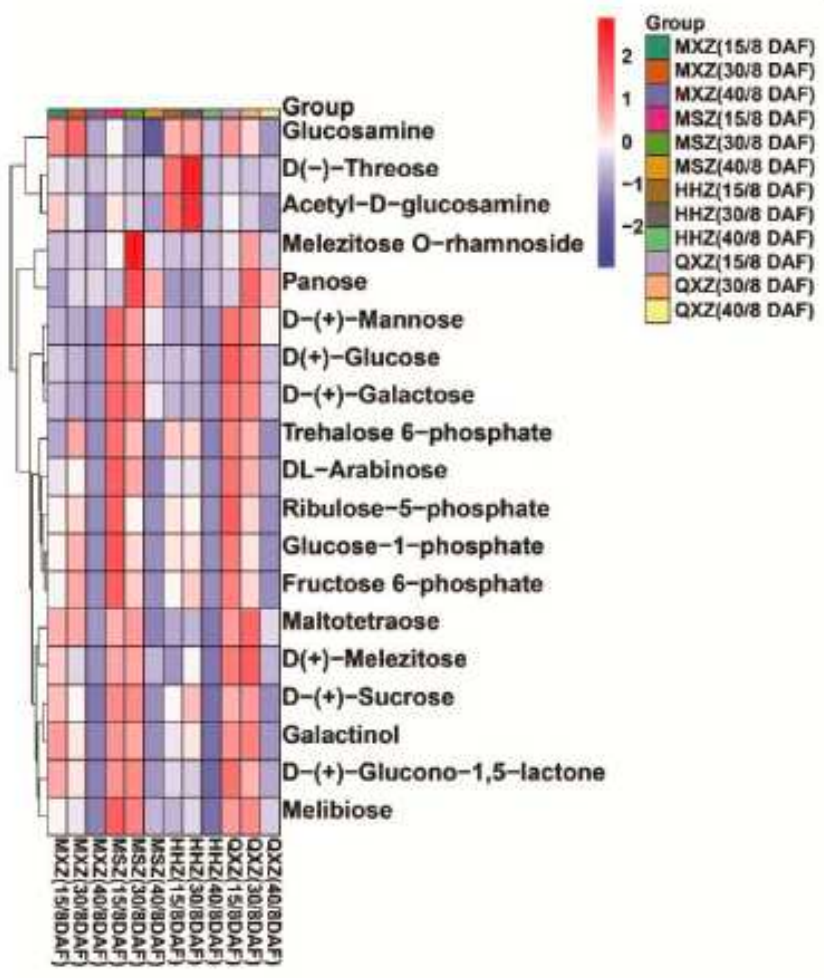

(c)

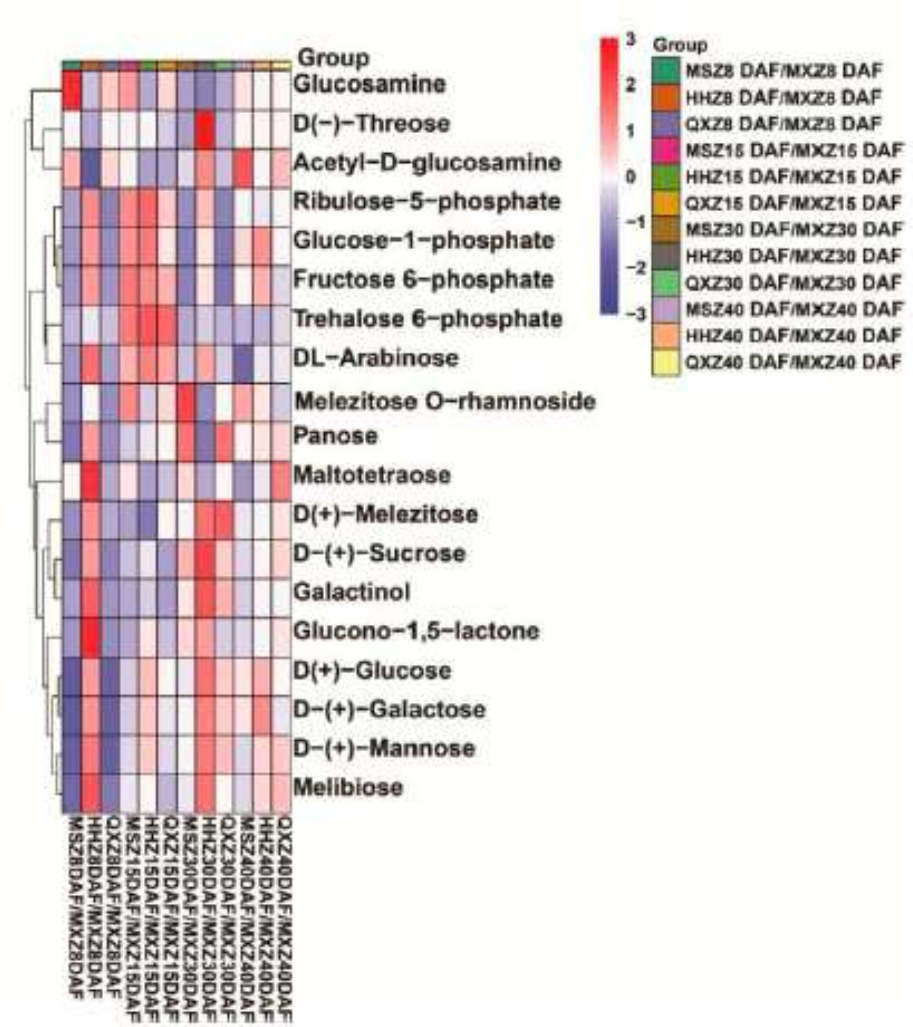

\section{Figure 2}

(a) Changes in metabolites mapped to the starch biosynthesis pathway in the four rice cultivars at 8,15 , 30, and 40 DAF. Abbreviations: UPDG, uridine diphosphate glucose; G-6-P, Glucose-6-phosphate; F-6-P, fructose-6-phosphate; G-1-P, Glucose-1-phosphate; (b) and (c) Heatmap of carbohydrate metabolite changes in the rice grains at $8,15,30$, and 40 DAF among the four cultivars (b) or compared with MXZ (c). Ratios of fold changes are given by shades of red or blue according to the scale bar. Data represent 
the mean values of three biological replicates for each cultivar and time point. For full metabolite names, refer to Table S1.

(a)

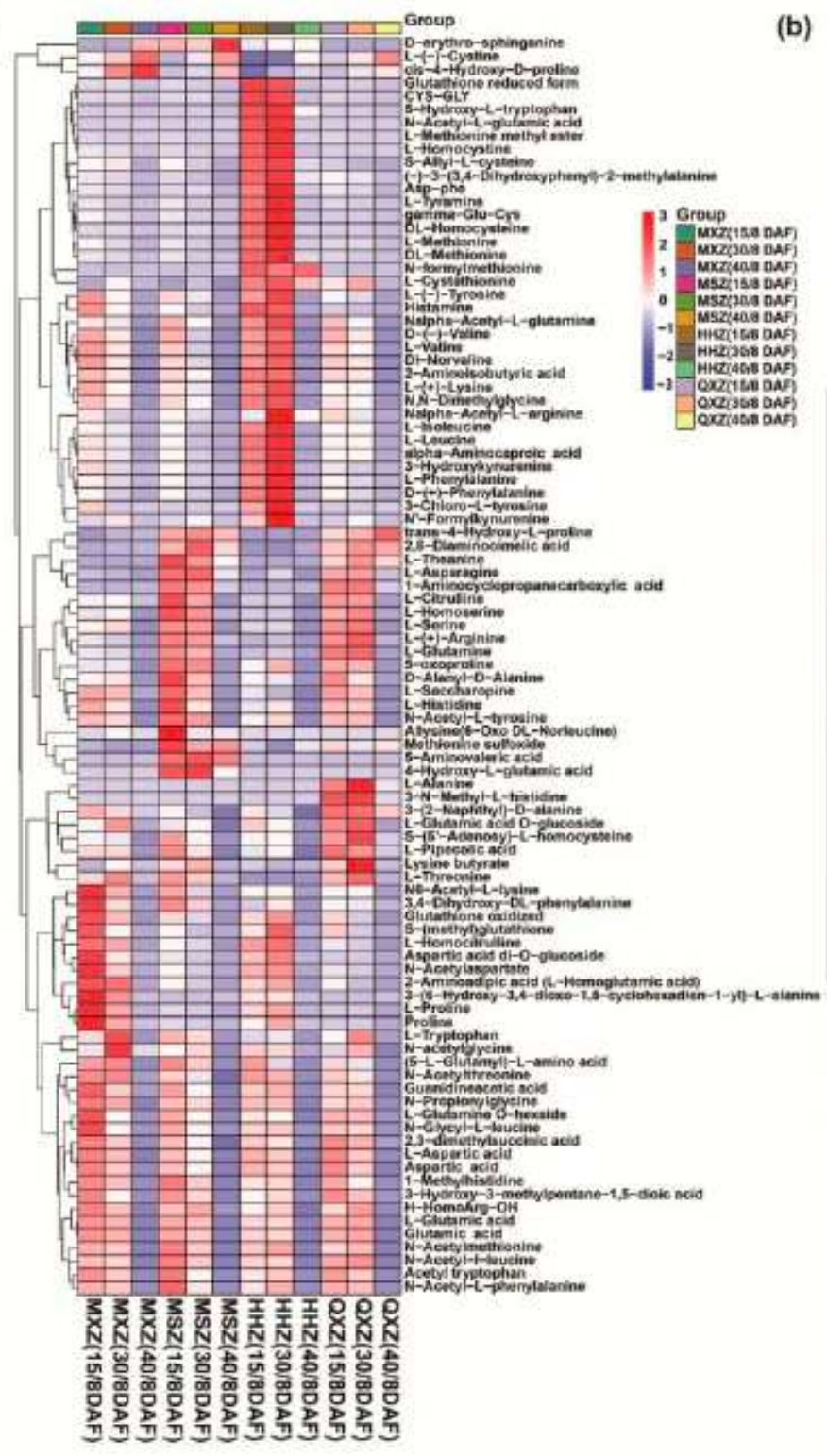

(b)
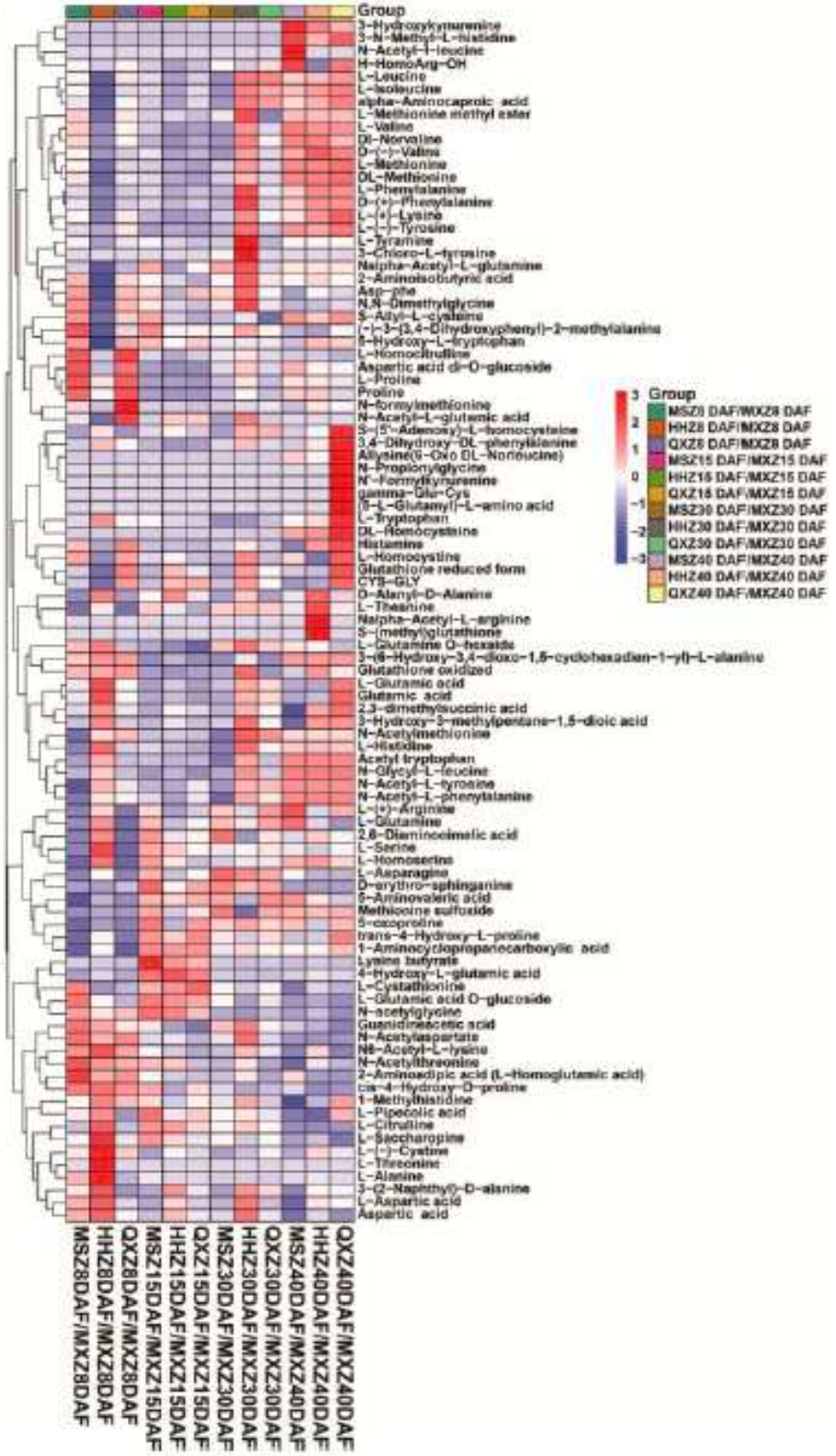

\section{Figure 3}

Heatmap of the changes in amino acids and derivatives in the rice grains at 8, 15,30, and 40 DAF among the four cultivars (b) or compared with MXZ (c). Ratios of fold changes are given by shades of red or blue according to the scale bar. Data represent the mean values of three biological replicates for each cultivar and time point. For full metabolite names, refer to Table S1. 

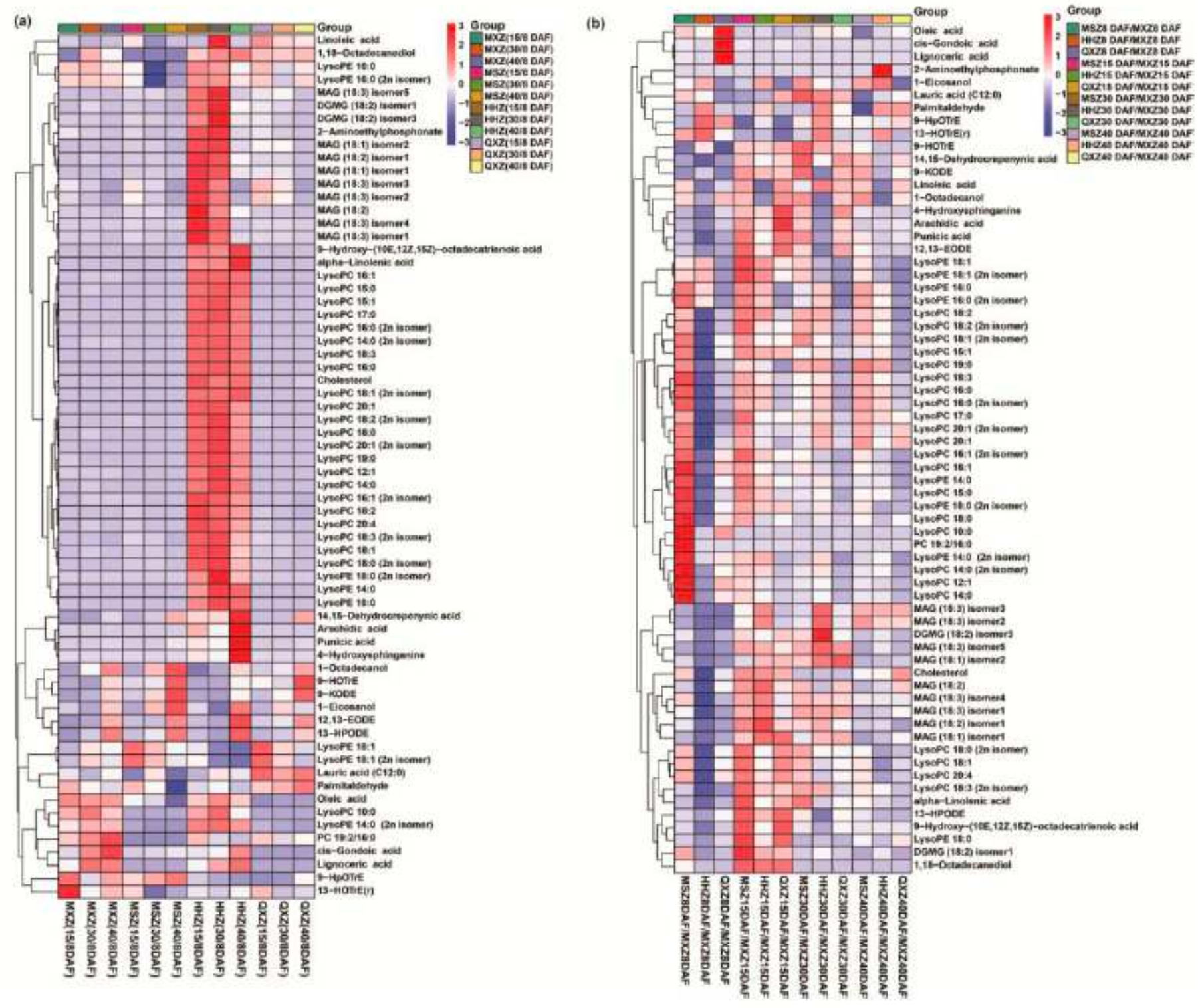

Figure 4

Heatmap of lipid metabolite changes in the rice grains at 8, 15, 30, and 40 DAF among the four cultivars (b) or compared with MXZ (c). Ratios of fold changes are given by shades of red or blue according to the scale bar. Data represent mean values of three biological replicates for each cultivar and time point. For full metabolite names, refer to Table S1. 
(a)

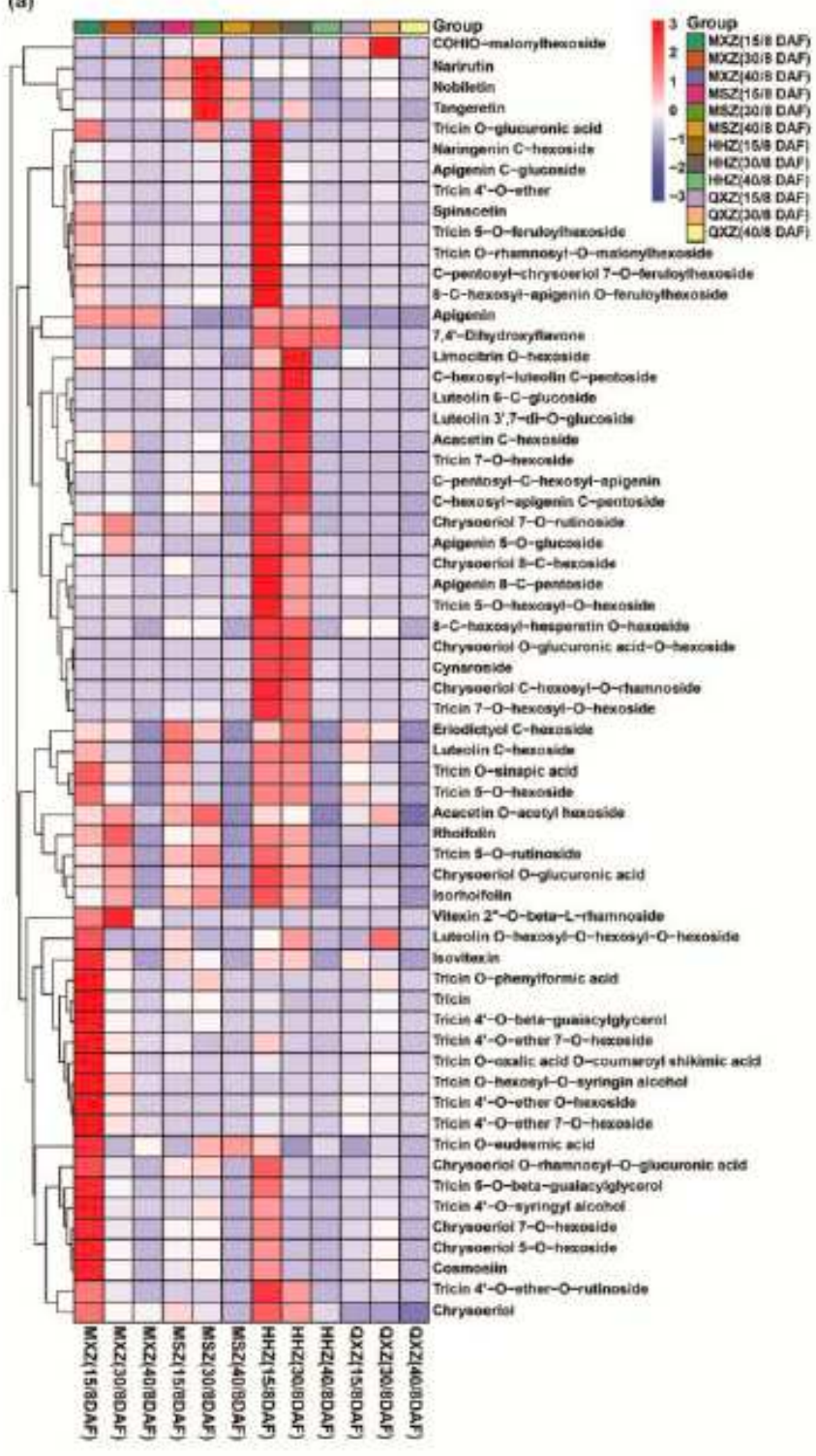

(b)

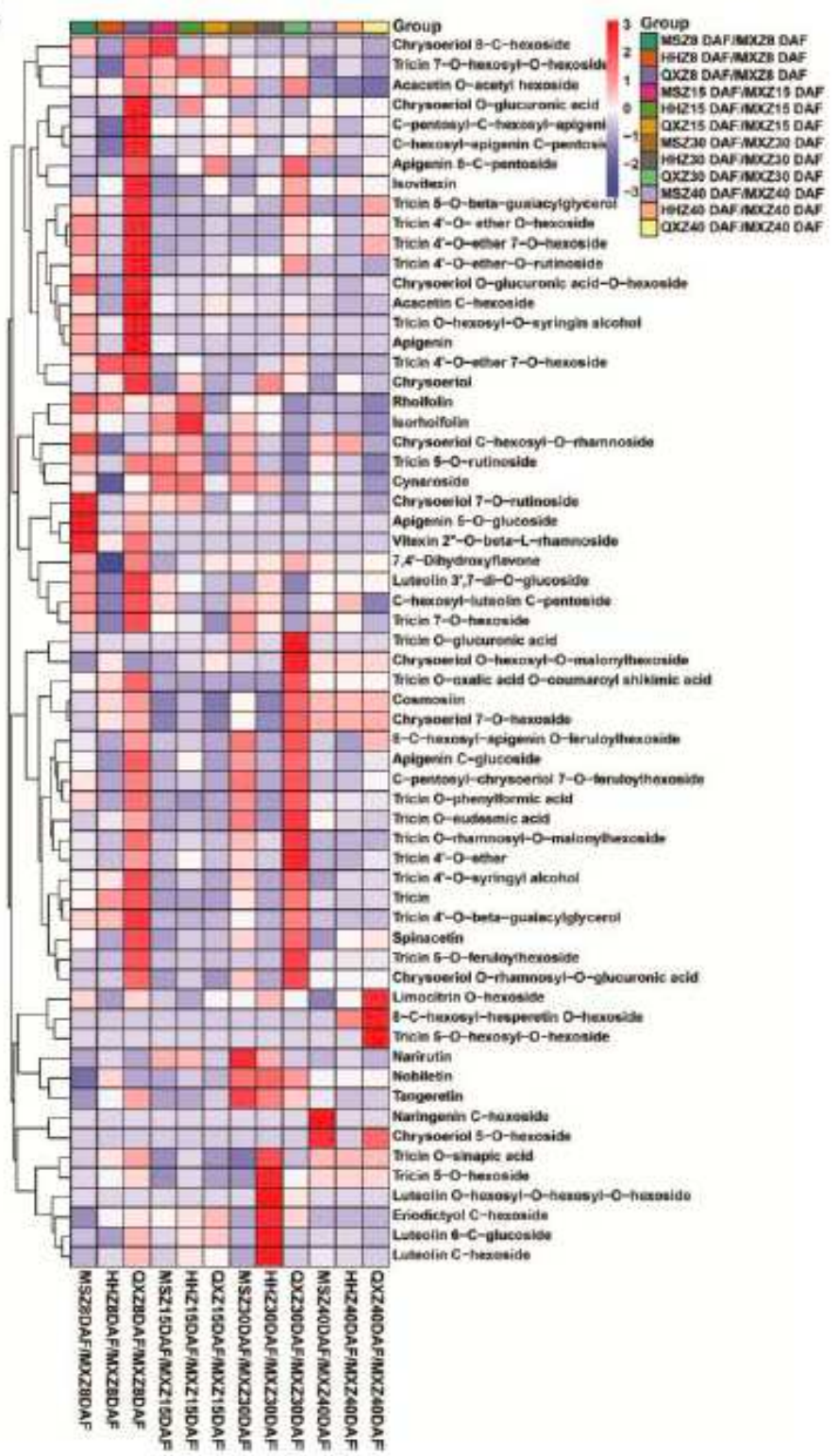

Figure 5

Heatmap of flavones metabolite changes in the rice grains at 8, 15,30, and 40 DAF among the four cultivars (b) or compared with MXZ (c). Ratios of fold changes are given by shades of red or blue according to the scale bar. Data represent mean values of three biological replicates for each cultivar and time point. For full metabolite names, refer to Table S1. 
(a)

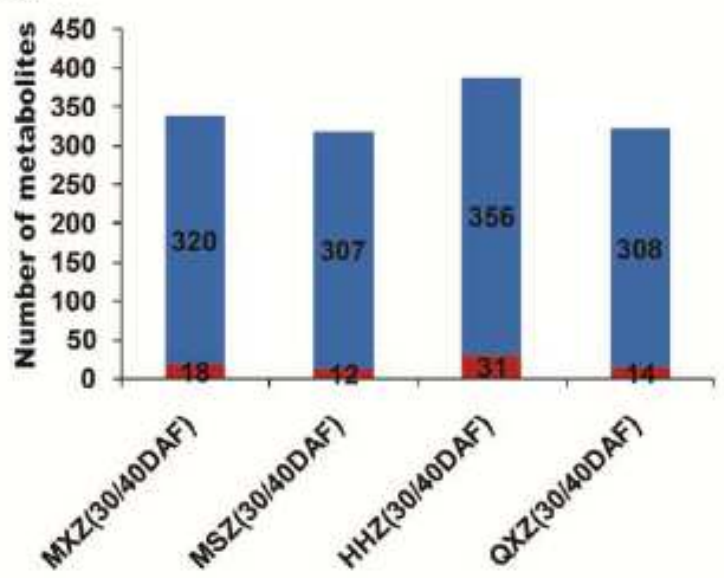

(c)

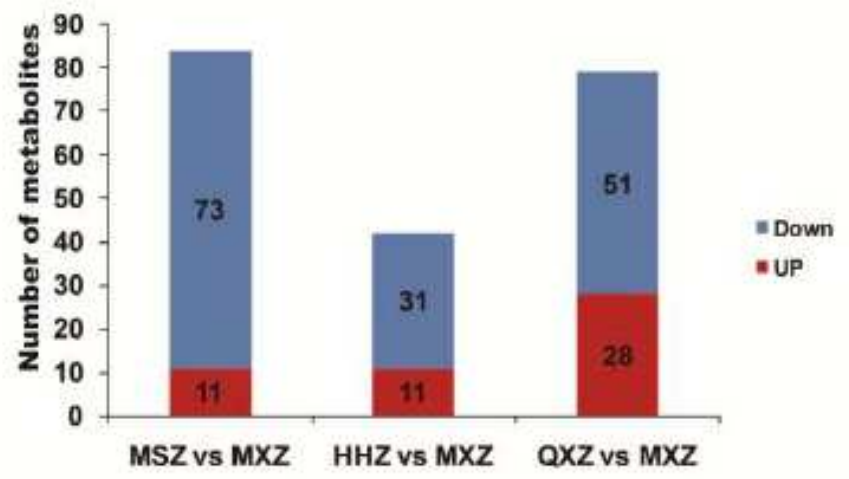

(b)

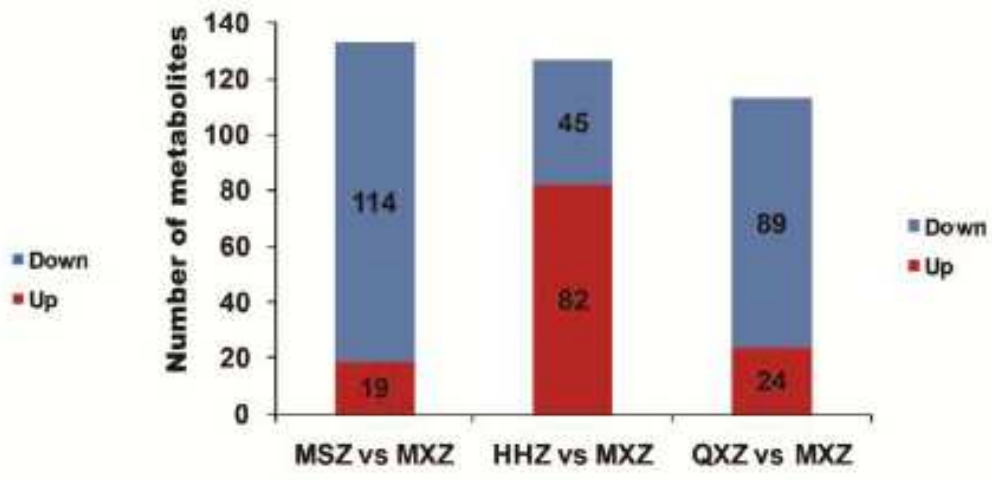

(d)

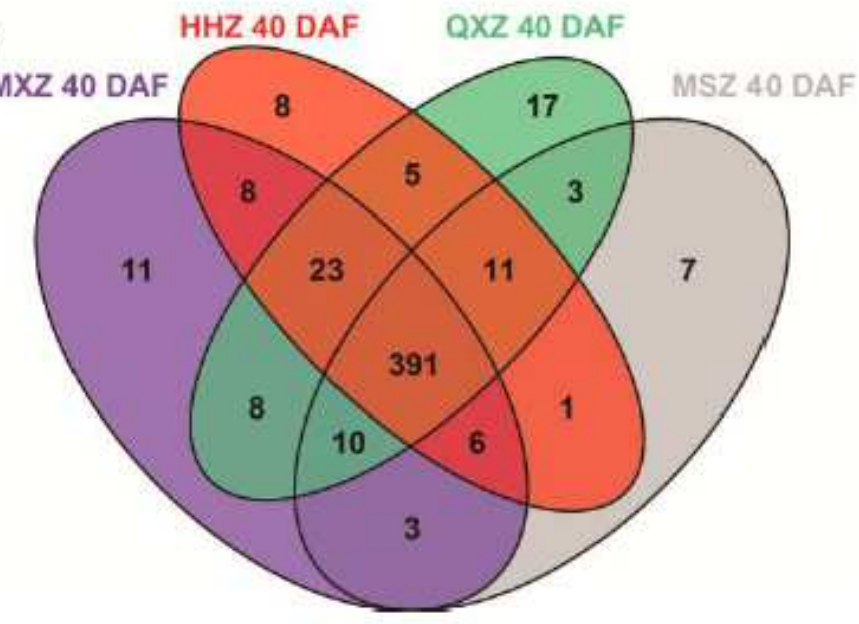

\section{Figure 6}

(a) Number of metabolites with differential changes $\geq 2$-fold and $<0.5$-fold $(P<0.05)$ in the four cultivars during the post-harvesting process (from 30 to $40 \mathrm{DAF}$ ). Number of metabolites with differential changes in MSZ, HHZ, and QXZ compared with MXZ at 30 DAF (b) and 40 DAF (white rice) (c). (d) Venn diagram of significantly changed metabolites in the four cultivars at $40 \mathrm{DAF}$ (white rice).

\section{Supplementary Files}

This is a list of supplementary files associated with this preprint. Click to download.

- SupplementaryMaterial.rar

- SupplementalFigures.pdf 